19 Revue d'histoire du XIXe siècle

Société d'histoire de la révolution de 1848 et des

révolutions du XIXe siècle

$26 / 27 \mid 2003$

Varia

\title{
Les débuts de la médecine catholique en France
}

La Société médicale Saint-Luc, Saint-Côme et Saint-Damien (1884-1914)

Hervé Guillemain

\section{(2) OpenEdition}

Journals

Édition électronique

URL : https://journals.openedition.org/rh19/747

DOI : 10.4000/rh19.747

ISSN : $1777-5329$

Éditeur

La Société de 1848

Édition imprimée

Date de publication : 1 décembre 2003

Pagination : 227-258

ISSN : 1265-1354

\section{Référence électronique}

Hervé Guillemain, "Les débuts de la médecine catholique en France », Revue d'histoire du XIXe siècle [En ligne], 26/27 | 2003, mis en ligne le 19 février 2008, consulté le 28 février 2022. URL : http:// journals.openedition.org/rh19/747 ; DOI : https://doi.org/10.4000/rh19.747

Ce document a été généré automatiquement le 28 février 2022.

Tous droits réservés 


\title{
Les débuts de la médecine catholique en France
}

La Société médicale Saint-Luc, Saint-Côme et Saint-Damien (1884-1914)

\author{
Hervé Guillemain
}

1 "Il règne en certains milieux une idée préconçue: on se persuade que le savant catholique n'est qu'un soldat au service de la foi, et que la science ne peut être en ses mains qu'une arme pour la défense de son credo. La meilleure réponse à faire à ce préjugé, c'est de cultiver la science pour elle-même, sans y chercher directement aucun intérêt apologétique. La profession de la foi chrétienne ne doit pas faire obstacle aux généreuses initiatives, même aux hardiesses du savant. L'erreur peut être la devancière et est souvent la compagne de la vérité ${ }^{1}$.

2 La position de Mgr Mercier au Congrès catholique de Malines en 1891, donne un aperçu des débats qui agitent le monde catholique à la fin du XIX ${ }^{\mathrm{e}}$ siècle sur la nature de la «science catholique ». L'histoire sociale et intellectuelle de cette figure professionnelle émergeante - celle du médecin catholique - se trouve au cœur des tensions d'une époque et d'un affrontement culturel ancien entre la science et le religieux; elle peut être insérée aussi dans les derniers développements historiographiques de l'histoire de la médecine, qui mettent l'accent sur la pluralité des voies qu'emprunte le processus de médicalisation et de façon générale, sur les marges de la médecine officielle ${ }^{2}$.

3 L'existence de la Société médicale Saint-Luc, Saint-Côme et Saint-Damien, organisation des médecins catholiques fondée en 1884, n'a été que succinctement évoquée dans les travaux universitaires, la médecine chrétienne restant par ailleurs peu explorée ${ }^{3}$. L'histoire de cette société, qui est une des premières à organiser l'action de catholiques en milieu professionnel, présente plusieurs intérêts : des archives originales inédites sur une longue durée, appuyée par la publication régulière d'un bulletin; une date de création, 1884, contemporaine d'un regain d'attrait du monde catholique et romain pour la science dans un contexte théologique offensif et dans un contexte politique conflictuel; un groupe d'hommes agissant dans un milieu a priori hostile ou méfiant, agnostique ou anticlérical. Le nombre de membres de la Société Saint-Luc, entre 150 et 1200 sur la période étudiée (1884-1914), la publication de leurs travaux, fait sortir de la 
marginalité ces médecins catholiques. L'image populaire du médecin anticlérical a totalement gommé l'existence de ce médecin chrétien, et on doit s'interroger sur la place de ce praticien dans un processus complexe et inégal de médicalisation et de déchristianisation ${ }^{4}$. L'histoire sociale des médecins français a montré que le savoir médical n'était pas une production indépendante des savoirs populaires ou religieux, mais qu'au contraire, l'ensemble de ces savoirs pouvait avoir des sources communes ${ }^{5}$.

Peut-on parler à propos de la Société médicale Saint-Luc d'une tentative de formation d'une science médicale catholique ? Le concept de «science catholique » est antérieur aux années 1880 et s'applique d'abord aux questions historiques et bibliques ${ }^{6}$. Il s'agit de montrer le bon accord de la science et de la foi, en réaction à la science antichrétienne des Lumières. La réflexion médicale est intégrée à ce projet, essentiellement théologique, comme on peut le constater dans certains volumes de L'Encyclopédie théologique de Migne (1844-1873), dans la revue d'apologétique scientifique dirigée par l'abbé Jaugey, La Science catholique (1886), ou encore dans les Congrès scientifiques organisés par Mgr d'Hulst (1888-1900) 7. Dans cette optique, et parfois sous l'impulsion néo-thomiste romaine, les savants catholiques se placent au service de la foi et de la théologie dans le domaine qui est le leur. À la fin du XIX siècle, les questions abordées, qu'il s'agisse de l'évolutionnisme ou de l'hypnotisme par exemple, dérivent toujours d'une interrogation théologique mais font appel à des connaissances plus précises: physiologie, sciences naturelles, médecine, psychologie. La science catholique conserve un versant apologétique mais les savants catholiques produisent un savoir scientifique original qui s'émancipe du cadre théologique.

5 L'histoire de la Société médicale Saint-Luc, Saint-Côme et Saint-Damien, de sa fondation à la Première Guerre mondiale, permet d'ouvrir quelques pistes de réflexion sur la nature de cette "science catholique ». Après s'être attaché à évoquer le moment de la fondation, on donnera un tableau sociologique de l'organisation, de ses fondateurs et de ses membres, puis on envisagera la répartition quantitative et l'évolution des thèmes de prédilection du groupe des médecins catholiques. À partir de ce socle, il s'agit d'examiner les rapports de ces médecins avec l'Église, Rome et les praticiens qui se réclament d'une approche laïque, mais aussi d'envisager la production d'un savoir médical catholique. Afin de clarifier le statut du médecin chrétien dans la société et dans l'Église, on évoquera l'exemple de l'intervention de la Société à Lourdes, lieu de mémoire des affrontements du temps. Il faut s'interroger en fin de compte sur la légitimité de l'emploi de la notion de «médecine catholique » et sur ses rapports avec deux conceptions de la science catholique, l'une, apologétique, destinée à confirmer la théologie et à contrecarrer les évolutions laïques de la société, l'autre plus autonome étant l'expression d'une science exercée par des catholiques.

La renaissance d'une confrérie de médecins (1884)

6 La Société médicale Saint-Luc, Saint-Côme et Saint-Damien est fondée, le 27 septembre 1884, par un chirurgien du Mans, le docteur Jules Le Bêle (1820-1903). Elle est alors le fruit indirect d'une souscription destinée à la réalisation d'une chapelle dédiée aux saints médecins, au sein du Sacré-Cœur de Montmartre, lancée conjointement en juin 1883 par quelques médecins catholiques réunis en comité et par l'évêque du Mans, Mgr d'Oultremont. Celle-ci rassemble les dons de cinq cent quatre-vingt-quinze médecins français, cent vingt-quatre étudiants en médecine, quatre-vingt-deux pharmaciens, cent cinq familles de médecins et quatre cent vingt et un anonymes. Le succès de cette souscription incite à pérenniser la société. Le choix du Mans, comme lieu de la 
fondation, est tributaire de l'impulsion décisive donnée par le docteur Jules Le Bêle, une des grandes figures de la médecine chrétienne de la fin du siècle. Praticien dans la capitale sarthoise depuis 1845 , homme d'action et de réseau, il est issu d'une famille de médecins et de prêtres, et préfère une position de notable local à la carrière scientifique parisienne que son intégration dans le laboratoire de Claude Bernard pouvait lui faire espérer ${ }^{8}$. Ni érudit, ni grand novateur en matière médicale, il est avant tout un fervent catholique attaché à défendre la société et la morale chrétiennes. Le Bêle contacte les médecins catholiques de ce réseau informel de dévotion au SacréCœur, et forme le premier comité de la Société, comité fondateur largement dominé par les médecins de l'Ouest et qui comporte, par le hasard de la réunion, deux Bénédictins de Solesmes ${ }^{9}$ : Dom Couturier, seul à ne pas être médecin, est ainsi amené à prononcer le discours de fondation de la Société médicale Saint-Luc, Saint-Côme et Saint-Damien.

7 Les textes manifestes publiés dans les premières années de la Société permettent de définir sa philosophie: la Société médicale Saint-Luc est-elle un syndicat ou une confrérie ? Se donne-t-elle pour but de copier les sociétés savantes ou bien répond-elle essentiellement à des visées apologétiques? La loi sur les syndicats professionnels est votée le 21 mars 1884, six mois avant la fondation de la Société, mais la société de médecins catholiques est très éloignée du syndicalisme dans son esprit comme dans ses pratiques ${ }^{10}$. L'évolution de l'organisation vers la défense professionnelle est un débat récurrent au sein de la Société, débat toujours tranché par la négative avant 1914, au bénéfice du maintien de l'unité des membres : «des hommes de même profession, qui se réunissent d'eux-mêmes par le désir simplement chrétien de faire leur salut et de se sanctifier ${ }^{11}$. En suivant Dom Couturier, on pourrait faire de la Société Saint-Luc un des prémices de la sectorisation de l'action catholique, l'objectif étant de christianiser le monde médical, réputé fortement hostile au religieux. Le projet spirituel individuel de la Société, réservée aux seuls membres catholiques du corps médical, est opposé aux fins matérielles collectives du syndicat. Le modèle structurel de la Société médicale est à chercher du côté des sociétés Saint-Vincent-de-Paul et des anciennes confréries, son mode de recrutement étant restreint et fondé sur la cooptation.

Scientia et fides, les principes fondateurs de la société médicale catholique

8 L'esprit de la fondation est donc celui d'une refondation, assez proche de la tentative avortée du restaurateur de l'ordre dominicain, Henri Lacordaire, en 1840, dans le souci de concilier science, apologétique et pratique ${ }^{12}$ :

«Art. 4 : La Société a pour but : $1^{\circ}$ La sanctification de ses membres dans l'exercice de la médecine par l'union de leur foi et la conformité de leur conduite aux règles et aux enseignements de la Sainte-Église, par la charité réciproque, base de la vraie

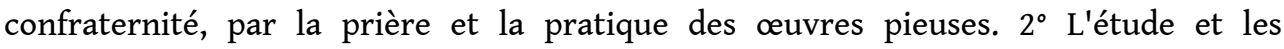
recherches de la science d'accord avec les lumières de la foi, en vue d'accroître constamment le savoir et l'expérience du médecin. $3^{\circ}$ Les travaux apologétiques destinés à défendre la vérité contre les théories opposées au véritable esprit scientifique, tel que l'a défini le Concile du Vatican. La Société appréciant l'importance capitale de l'enseignement médical chrétien déclare s'unir par les liens les plus étroits aux facultés ou écoles soit officielles, soit libres qu'elle reconnaîtra capables d'assurer cet enseignement. Elle encourage toutes les publications médicales conformes à la doctrine catholique ${ }^{13}$. 
10 Dans la nouvelle Société, les médecins sont unis par une même dévotion (dont le centre spirituel est Montmartre), par des pratiques religieuses communes (messe mensuelle pour les membres défunts, messe hebdomadaire de pénitence), par le pèlerinage (celui de Luzarches) et par l'invocation des saints médecins (celle des saints patrons chirurgiens syriens Saint-Côme et Saint-Damien) ${ }^{14}$. Dès les premiers temps pourtant, et comme l'indique le diplôme délivré aux adhérents, à la prière s'ajoute le travail scientifique, le savoir, scientia et fides. Ainsi le Docteur Ferrand, premier président de la Société, proclame-t-il en 1886 cette impérieuse nécessité : «Les pieuses pratiques ne sauraient supplanter la thérapeutique, un acte de foi ne peut tenir la place d'une solide démonstration scientifique ${ }^{15}$. Cette évolution est signifiée presque imperceptiblement : la Société Saint-Luc devient Société Médicale Saint-Luc en 1888. La pratique régulière et les actions de prière, imposées par les statuts, apparaissent en souffrance dans certains comités régionaux et les rappels à l'ordre du comité directeur de la Société sont fréquents. La pratique des pèlerinages locaux est encouragée afin de pallier ces insuffisances, les dirigeants escomptant développer les actes de prière par la conjonction des réunions professionnelles, savantes et religieuses ${ }^{16}$. Les travaux scientifiques et les discussions sont développés principalement dans les grands comités urbains (Paris, Lyon, Lille), le religieux prenant le pas dans les petits comités de province et surtout dans l'Ouest. Si la régularité de la pratique religieuse peut entrer en ligne de compte dans ce clivage, il faut aussi imputer à la charge des médecins de campagne, à leur éloignement des lieux de réunion, la faible animation scientifique de certains comités de province. En 1890 encore, les études scientifiques sont globalement restreintes et l'assistance mutuelle et professionnelle est inexistante entre les membres, ce qui fait dire au président: «Le lien religieux peut seul nous maintenir étroitement unis en dehors des grands centres où l'élément et l'attrait scientifiques sont étouffés par l'active et laborieuse mission dévolue à nos confrères à la campagne ${ }{ }^{17}$. En 1897, le tournant scientifique paraît consommé, et afin de faire croître les effectifs de l'association, on souhaite gommer l'image trop dévote du groupe médical catholique. La mutation, peu affichée publiquement, est néanmoins réelle et significative du dépassement du modèle confraternel, qui subsiste en héritage, vers un modèle de société savante tournée vers l'action catholique et l'apologétique offensive.

Le temps de la défense catholique contre l'irréligion scientifique

11 Cette vocation savante dans un cadre confessionnel ne peut être détachée d'une visée apologétique rendue nécessaire par les attaques anticléricales sévères des années $1880^{18}$ et incitée par Léon XIII par le biais de l'Encyclique Humanum Genus. Cinq mois avant la fondation de la Société (20 avril 1884), le texte pontifical invite tous les catholiques à lutter, avec leurs compétences, contre l'athéisme et l'emprise croissante $\mathrm{du}$ rationalisme ${ }^{19}$. La science, la médecine surtout, est en première ligne dans l'affrontement idéologique, et le Souverain Pontife allie son désir de reconquête des âmes à celui de l'investissement de la science par les fidèles. La Société médicale SaintLuc, reconstitution moderne de la confrérie médiévale Saint-Luc, sous le patronage, certes limité, de l'épiscopat ${ }^{20}$, répond point par point au souhait de Léon XIII. Le texte fondateur insiste sur cette allégeance précoce : «votre société, par ses statuts et son organisation, donne le véritable modèle que devront suivre, si elles veulent réussir, les corporations que le Très Saint Père recommandait ${ }^{21}$. 

notamment dans le cadre de l'Institut catholique de Paris ou de la Faculté de Lyon. Des théologiens viennent prendre part aux discussions scientifiques, notamment sur les questions relatives à la psychologie et à l'hypnotisme, sujets brûlants qui nécessitent une collaboration entre médecins et ecclésiastiques ${ }^{29}$. Le conseil central relaie la requête des médecins du comité de Marseille auprès de l'épiscopat afin d'interdire effectivement l'exercice illégal de la médecine par certains prêtres peu scrupuleux: c'est le signe de la persistance d'un problème considéré trop hâtivement comme réglé dans le courant $\mathrm{du} \mathrm{XIX}^{\mathrm{e}}$ siècle ${ }^{30}$. Pourtant la pression de quelques prêtres pour l'adjonction systématique d'un théologien dans chaque comité n'a pas de suite, sans que l'on sache pour quelles raisons. Ces relations pacifiées avec l'Église de France ne doivent pas faire oublier la méfiance dont sont entourés ces médecins catholiques dans certains cercles cléricaux, médecins que l'on préférerait organisés dans le cadre de la Société Saint-Vincent-de-Paul. Les procès-verbaux des réunions du conseil central de la Société Saint-Luc témoignent du désir d'autonomie des médecins face à l'Église, ce qui justifie l'appel au magistère romain, comme celui du docteur Guéniot : «L'opinion de tel ou tel ecclésiastique, si respectable soit-il, n'a aucune valeur devant une décision du Saint-Office ${ }^{31}$. Certains n'hésitent pas, il est vrai dans le secret des débats à huis clos, 
à poser les bases d'une autonomie des médecins vis-à-vis de l'Église ; ainsi le docteur Alibert, membre du comité de Paris, s'interroge en 1898 :

«Avec les données scientifiques nouvelles, ne serait-il pas bon de réviser les règles de l'Église, basées sur des connaissances anciennes et souvent erronées. Notre but doit être d'éclairer l'Église sur les progrès scientifiques qui s'accomplissent ${ }^{32}$.

Si des contacts sont noués avec les Conférences Saint-Vincent-de-Paul, avec les patronages ou les cercles ouvriers, l'essentiel de l'effort d'ouverture des médecins porte vers les étudiants en médecine. Dès 1886, les étudiants internes des hôpitaux de Paris, ceux du Cercle du Luxembourg ${ }^{33}$ et surtout ceux des Conférences Laënnec sont invités à se joindre à leurs pairs plus expérimentés, lors des assemblées générales et des fêtes patronales. Le souci d'associer les étudiants catholiques est immédiat, non seulement dans une optique formatrice mais surtout dans le but de promouvoir l'adhésion à la Société Saint-Luc chez les jeunes médecins ${ }^{34}$. Les Conférences Laënnec, qui ont pris le nom du médecin chrétien le plus réputé au XIX ${ }^{\mathrm{e}}$ siècle, René Laënnec (1781-1826) ${ }^{35}$, jouent un rôle croissant dans l'organisation des jeunes médecins. Fondées par les pères jésuites en 1875 afin de préparer les étudiants aux concours de l'internat et des hôpitaux, elles sont dirigées par le père Hubin à leur origine, mais sont, dès 1882, investies par de jeunes médecins qui jouent un rôle au sein de la Société Saint-Luc ${ }^{36}$. Les conférences comptent cent trente étudiants en 1889 , près de deux cents en 1895, près de trois cents en 1911. La déontologie professionnelle fait l'objet d'une attention permanente dans la formation des jeunes gens, règles édictées dans un Mémento du médecin catholique édité en 1911, qui met l'accent sur la nécessité pour le médecin de parfaire sa science dans une optique de dévouement au malade, de se sacrifier lors des épidémies, de respecter le secret médical, de contrer les influences néo-malthusiennes, d'avoir de saines prétentions financières ${ }^{37}$. Si les Conférences Laënnec et la Société Saint-Luc sont différentes par l'esprit qui les anime, les membres des deux organisations médicales catholiques ne cessent de renforcer leurs liens, qui deviennent étroits avant 1900 .

Les membres de la Société Saint-Luc : éléments statistiques et géographiques.

L'étude des effectifs de la Société Saint-Luc (graphique A) permet de dégager trois phases dans son évolution, entre 1886 et 1914. Peu après la création de la Société (1886-1890) se produit un recrutement rapide, sans publicité, par mobilisation des réseaux existants. Durant cette phase, le groupe des médecins catholiques passe de cent soixante membres à près de cinq cents. Dans un second temps (1890-1904), le recrutement se fait plus laborieux, puisque la moyenne annuelle passe de cent vingt adhésions par an à moins de vingt. Puis dans une troisième phase (1905-1911), la croissance du recrutement redevient forte, sur un rythme de soixante adhésions annuelles. La Société Saint-Luc dépasse le millier d'adhérents en 1910 et avoisine 1200 adhérents avant la guerre, même si point alors une baisse des effectifs, qui ne fait que s'accentuer lors du conflit, les pertes étant importantes, surtout chez les jeunes médecins. Il faut attendre le début des années vingt pour voir les effectifs de la Société dépasser 1200 adhérents. En 1927, la Société Saint-Luc compte environ 1500 membres. Ce succès est ambivalent, car si l'on rapporte les effectifs de la Société à l'ensemble des médecins français, on constate une stabilité relative autour de 5 à $6 \%$. La Société SaintLuc comprend donc une partie non négligeable du corps médical, mais certainement pas l'ensemble des médecins catholiques de France - seulement une majorité active ${ }^{38}$. 


\section{Ewohtion des enectis de ba Société SaintLuc (18ab6-1914)}

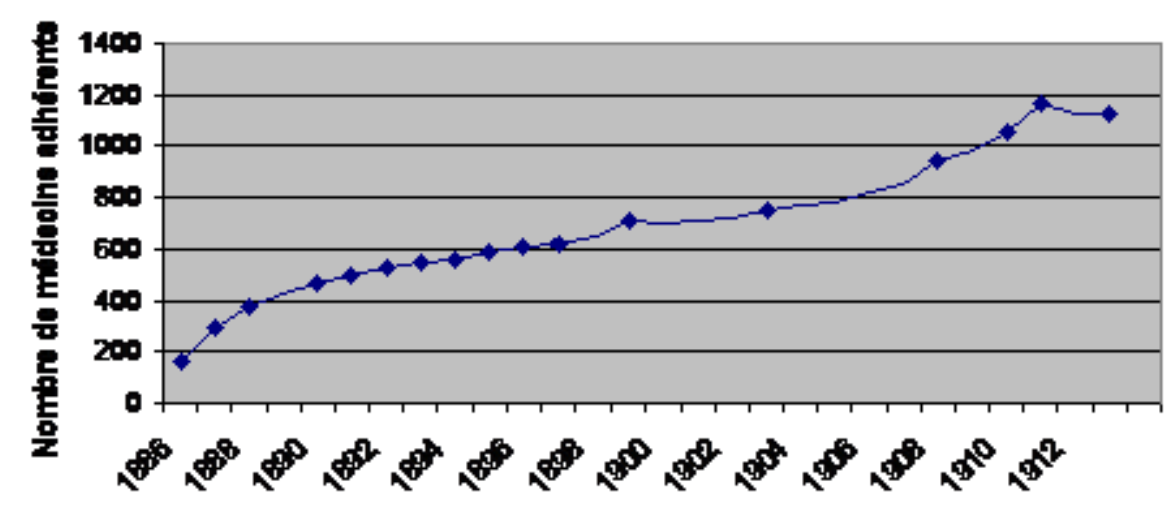

Les données chiffrées sont tirées principalement des annuaires de la Société. Les chiffres sont ceux des adhérents et des membres étrangers. Les points indiqués sur le graphique sont ceux pour lesquels on dispose de données fiables et comparables aux précédentes.

La croissance des effectifs impose aux médecins catholiques une organisation en comités régionaux et locaux, dont l'activité est décentralisée vis-à-vis du conseil central parisien, organe décisionnaire. Cette décentralisation perdure malgré des tensions récurrentes entre le centre et les périphéries et les difficultés liées à la représentation insatisfaisante des médecins de province. Ainsi, en 1887, les comités lillois et rennais tentent-ils de se réunir en dehors des institutions centrales parisiennes. Face à la menace d'éclatement, l'assemblée générale du 21 octobre 1888 confère au conseil central le droit de créer des comités locaux (dont la création peut paraître une stratégie de division), tandis que les comités trop indépendants sont tancés. Le comité de Paris, qui est aussi le conseil central de la Société, mène à partir de 1895, une politique de centralisation accrue, qui entraîne une réaction des comités de Lille, de Lyon, du Centre et de Marseille. Après 1900, le docteur Féron-Vrau tente une conciliation, en associant les comités de province au conseil central, mais le pouvoir des médecins parisiens reste fort, surtout dans le comité de rédaction du bulletin.

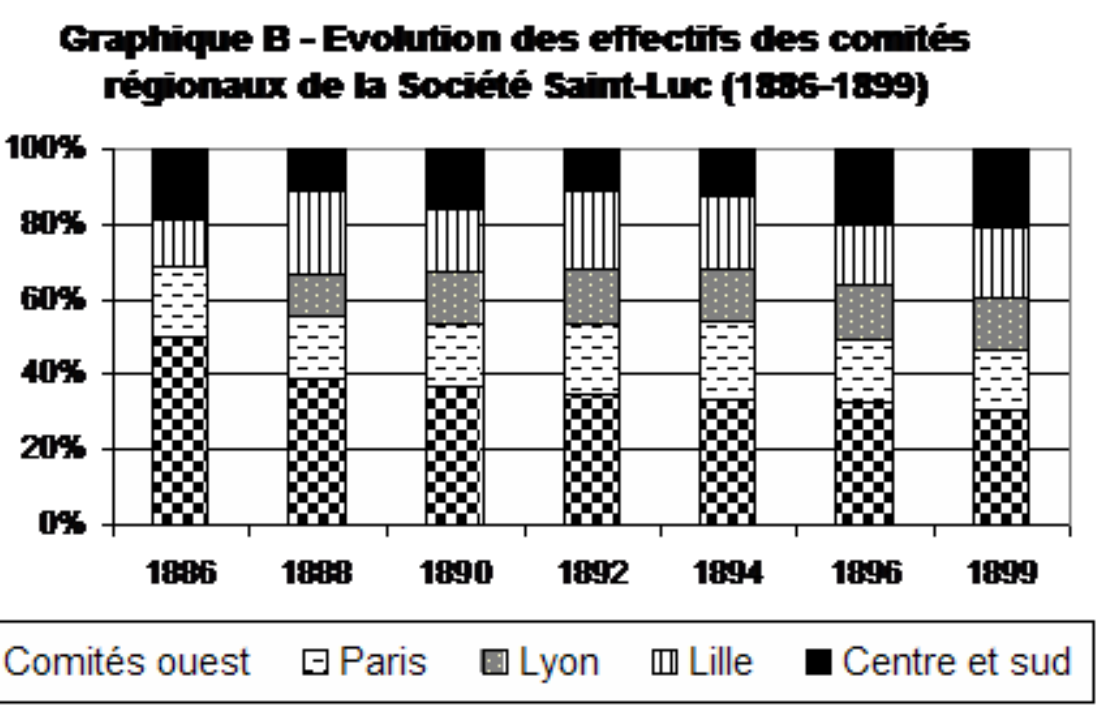


21 Les comités les plus actifs dès la fondation sont, avec celui de Paris, les comités de l'Ouest (Rennes, Rouen, Le Mans), avant que ne s'affirment les comités des grandes villes, tels que ceux de Lille et de Lyon, appelés à devenir les plus importants quelques années plus tard (graphique B). Si les comités de l'Ouest, qui correspondent principalement aux régions catholiques pratiquantes, dominent les premières années, ils atteignent rapidement un étiage maximal, tandis que les comités urbains drainent un nombre de médecins en rapport avec la clientèle abondante de ces régions et avec la présence de lieux de formation médicale et d'universités catholiques. Malgré la volonté d'équilibrer la géographie des décideurs, la direction de la Société se déplace progressivement des médecins des villes de l'Ouest, vers les médecins lillois et lyonnais, ceux de Paris ayant une prépondérance constante ${ }^{39}$. Les médecins de cabinet des villes moyennes sont peu à peu remplacés par les médecins hospitaliers des grandes villes qui ont une activité de publication et une reconnaissance extérieure plus forte. On peut noter la relative marginalité des médecins du Sud, du Centre et de l'Est, qui ne s'estompe que partiellement avant la guerre. En 1899, un tiers des médecins adhérents font partie d'un comité de l'Ouest, près d'un médecin sur cinq est parisien, un sur cinq lillois, un sur six lyonnais. La géographie de la médecine catholique ne se modifie guère après $1900^{40}$.

Les médecins catholiques fondateurs de la Société Saint-Luc : éléments de sociologie

Qui sont ces médecins qui s'affirment comme catholiques dans un milieu professionnel réputé anticlérical et agnostique? Pour définir leur profil, il a été choisi de se concentrer sur le groupe des fondateurs et sur celui des principaux dirigeants, entre 1884 et 1904, soit une vingtaine de praticiens. On peut d'abord constater la prégnance du milieu familial dans l'exercice de la médecine, constatation qui n'est pas nouvelle, mais qui peut être confirmée dans le cas des médecins catholiques. Les confrères de Saint-Luc héritent souvent d'une tradition familiale médicale et religieuse, et transmettent à leurs enfants cette vocation de la médecine catholique. Exceptés le fondateur lui-même, Jules le Bêle, né en 1820, et le plus jeune des dirigeants, le docteur Pasteau, né après 1870, l'ensemble des membres fondateurs de la Société Saint-Luc appartient à une génération relativement homogène, née entre 1830 et 1855, et formée entre 1850 et 1880, à une époque durant laquelle le médecin prend une place sociale majeure ${ }^{41}$. C'est le temps de l'essor des laboratoires et de la pratique expérimentale, symbolisé par les travaux de Claude Bernard et de Louis Pasteur. La science se détache progressivement des préoccupations métaphysiques, même si la période du Second Empire paraît favorable à l'expression d'une médecine chrétienne.

Dans le groupe déterminé, il est possible de distinguer les internes des hôpitaux de Paris, sur-représentés par rapport au total des adhérents, et les médecins formés dans les écoles de médecine et les facultés de province qui constituent la majeure partie des membres. La carrière professionnelle de ces médecins est plus homogène que leur formation, puisque la plupart de ces médecins exercent en hôpital et enseignent dans les facultés de médecine. Deux membres directeurs ont des responsabilités à la Faculté libre de médecine de Lille ${ }^{42}$. Si la formation est largement parisienne, la carrière est largement provinciale : trois quarts des hommes du groupe ${ }^{43}$ exercent hors de Paris l'essentiel de leur vie professionnelle. Les domaines d'exercice, qui ne sont pas réellement des spécialités ${ }^{44}$, s'avèrent également assez homogènes dans ce groupe et marqués fortement par l'appartenance confessionnelle. En effet, parmi ces médecins on compte un nombre important de praticiens de la chirurgie obstétrique, "spécialité » 
fréquemment choisie dans les milieux catholiques, un groupe conséquent de neurologues et aliénistes, auxquels peuvent s'ajouter quatre médecins praticiens de l'hypnotisme, ainsi qu'un groupe de spécialistes de la médecine pour enfants. Ce triple intérêt de la médecine catholique pour la naissance, les troubles psychiques et l'enfance perdure au XXe siècle.

Grâce à ces données, auxquelles il faut ajouter l'activité éditoriale, la production d'articles dans les revues scientifiques et/ou catholiques, la participation aux sociétés savantes, les contacts avec l'Église, on peut d'ores et déjà dessiner plusieurs profils assez différents de médecins catholiques. Sur vint-cinq biographies étudiées, on élabore une typologie en quatre ensembles. Les moins nombreux sont les médecins notables au cursus uniquement provincial, dans la France de l'Ouest essentiellement ; ces médecins sont en relation étroite et permanente avec l'épiscopat de leur diocèse, et ont des fonctions d'expertise pour les cas d'apparition ou d'exorcisme notamment. Anatole Bucquet et Gustave Regnault, présidents du comité de Laval et de Rennes, sont ainsi experts lors des phénomènes d'apparition de Pontmain en 1871. Ces praticiens, parmi les plus âgés de la Société, occupent les directions des comités des régions catholiques ferventes. Ils jouent un rôle dans les institutions charitables locales et même dans les conseils municipaux, ce qui leur confère une stature de notables. Ces hommes sont reconnus localement et uniquement dans les milieux catholiques, comme en témoigne la diffusion restreinte de leur production écrite. Jules le Bêle, fondateur de la Société Saint-Luc, est le meilleur exemple de ce premier type de médecins catholiques. Les comités locaux sont également dirigés par un second type de médecins qui, comme les premiers, ne se distinguent guère par leurs publications et leur activité scientifique. Ils ont une vocation d'érudit qui s'exerce sur des sujets historiques touchant de près au passé de la Société, ou évoquant l'évolution du corps médical régional. Ils sont membres d'académies scientifiques régionales mais n'appartiennent pas aux grandes sociétés médicales nationales. Contrairement aux premiers qui ont une implantation occidentale, ils proviennent surtout des comités du Sud (Marseille) et de l'Est (Besançon, Lyon). Ces médecins peu spécialisés sont le plus souvent confinés à la sphère régionale des responsabilités. Henri Alezais, président du comité de Marseille, dirige un laboratoire de physiologie régional et mène une activité d'historien érudit concernant la médecine phocéenne du XVIII ${ }^{e}$ siècle.

Le troisième type de médecins catholiques de la Société Saint-Luc est très nettement distinct des deux premiers. En effet, quatre médecins parmi les vingt-cinq du groupe directeur se distinguent nettement des autres par l'originalité de leur activité scientifique et par la qualité de leurs travaux novateurs. Leur stature nationale est reconnue à l'extérieur de la Société et hors du monde catholique. Ces hommes sont tous membres de sociétés savantes médicales non confessionnelles, parmi les plus influentes de leur spécialité. Ainsi Victor Parant, aliéniste toulousain, directeur du comité du SudOuest, est un membre important de la Société médico-psychologique et compte parmi les rédacteurs les plus réguliers des Annales médico-psychologiques, revue de référence des aliénistes, et des Annales de psychiatrie. Ses articles, publiés entre 1880 et 1885, montrent son apport original à la question de la responsabilité des aliénés et au maintien d'une part raisonnable dans la folie ${ }^{45}$. Ces praticiens collaborent aussi aux nombreuses revues catholiques généralistes qui vulgarisent les apports scientifiques pour les fidèles non initiés. Ainsi, Ange Ferrand, premier président de la Société SaintLuc, spécialiste de psycho-physiologie, novateur dans le domaine de l'aphasie, rédacteur d'une seconde édition de la célèbre Théologie morale des sciences médicales du 
père Debreyne ${ }^{46}$, publie-t-il ses travaux et des articles de vulgarisation sur les grands débats scientifiques des années 1880-1890 dans L'Univers aussi bien que dans les Annales de philosophie chrétienne ${ }^{47}$. Des revues scientifiques catholiques accueillent à partir des années 1880 les publications de ces médecins sur les sujets polémiques tels que l'hypnotisme, l'hystérie, les miracles de Lourdes par exemple. Le docteur Ferrand, qui reste le médecin catholique le plus actif de cette génération, investit les sociétés savantes laïques non confessionnelles et y prend des responsabilités fortes dans la dernière décennie du siècle ${ }^{48}$. Ses travaux font autorité au-delà du monde des médecins catholiques sur les questions concernant l'aphasie et la psychologie de la parole mais aussi celles des localisations cérébrales, débat scientifique capital de la fin du siècle ${ }^{49}$. Le premier président de la Société Saint-Luc est, outre un organisateur dynamique, une des figures de la médecine psycho-physiologique de son temps. On peut en dire autant de Joseph Grasset, dont les travaux ont encore plus d'ampleur hors des milieux catholiques. Honoré à Montpellier qui abrite sa statue, le professeur Grasset s'est fait une réputation dans les milieux de la psychiatrie et de la neurologie naissante et fait partie de comités de rédaction de grandes revues spécialisées ${ }^{50}$. Membre directeur du comité du Languedoc de la Société Saint-Luc, il participe à la fondation de la Société d'hypnologie, une société non confessionnelle. Dépassant les études physiologiques, hypnologiques et psychiatriques, ses travaux sur le cerveau s'enrichissent d'études ultérieures dans les domaines biologiques et philosophiques, qui le conduisent à formuler un système médico-philosophique conforme à son orientation catholique et à la tradition vitaliste de l'université montpelliéraine. On peut ajouter à ces exemples, et dans le même domaine, celui des maladies nerveuses, le cas de Jean Camus, psychiatre, membre de la Société Saint-Luc mais aussi des Conférences Laënnec ${ }^{51}$. Celui-ci travaille sous l'influence de Déjerine sur le concept de psychothérapie, en vogue au début du XX siècle ${ }^{52}$. Les Annales médico-psychologiques voient en lui, à sa mort en 1924, un grand praticien de la psychiatrie. Ces quatre médecins catholiques, qui ont des responsabilités au sein de la Société, ont dans les milieux médicaux une reconnaissance qui dépasse celle des autres; il est intéressant de noter que tous travaillent dans les domaines des maladies nerveuses et de la psycho-physiologie ${ }^{53}$.

Le quatrième type de médecins est le plus important en nombre et ses représentants sont les plus actifs dans la défense catholique militante. Ces praticiens, qui jouissent tous d'une fonction soit régionale soit nationale importante dans la Société, ont une activité scientifique qui est avant tout orientée vers une fin apologétique, sans que cela apporte forcément des éléments novateurs au savoir médical. Ces médecins interviennent ainsi dans les grands débats de société : délimitation de l'usage de l'hypnotisme dans les années 1880 par les docteurs Guermonprez et Desplats ${ }^{54}$, défense des miracles de Lourdes par les docteurs Boissarie et Le Bec, défense des congréganistes dans les hôpitaux publics par le docteur Gouraud, publication de manuels médicaux pour les missionnaires par le docteur Dauchez, défense d'une politique nataliste catholique par les docteurs Alibert, Delore et Dauchez. Ces médecins, qui représentent quarante pour cent de notre groupe directeur, investissent les institutions charitables locales (Saint-Vincent-de-Paul, Bureau de bienfaisance) et jouent un rôle capital dans les organisations de jeunes médecins, telles que les Conférences Laënnec. Le fonctionnement du Bureau des Constatations de Lourdes, qui atteste la véracité des guérisons, et dont l'histoire a déjà été en grande partie écrite ${ }^{55}$, est assuré par trois de ces hommes, les docteurs Boissarie, Desplats et Le Bec, assistés d'autres médecins de la Société Saint-Luc. L'enseignement de la Faculté libre de médecine de Lille est de leur 
ressort dans une université fondée par Camille Féron-Vrau, grand ordonnateur de la Société Saint-Luc au début du XXe siècle. Le champ et les méthodes d'action de ce dernier préfigurent déjà les grandes heures de l'Action catholique ${ }^{56}$. Les hommes de cette génération, nés pour la plupart après 1840 et plus jeunes en moyenne que les médecins des trois autres types, ont été formés pour certains dans le giron des Conférences Laënnec, ce qui explique leur goût pour l'apostolat laïc. L'exemple le plus probant est celui de Henri Dauchez (1853-1919), un des premiers membres des Conférences Laënnec, après leur création par les Jésuites en 1875, qui en devient un des piliers, avant d'intégrer la Société Saint-Luc.

Une apologétique médicale : les thèmes de prédilection des médecins catholiques

Les médecins catholiques se distinguent-ils des autres quant à leur thème de prédilection? Les hommes de la Société Saint-Luc, bien avant que le bulletin ne publie leurs travaux, ont une activité conférencière importante au sein des comités régionaux. Il est possible de recenser ces conférences grâce aux archives de la Société et de classer thématiquement un ensemble de trois cent cinquante sujets donnés entre 1884 et 1904 (graphique C), et un second ensemble de cent soixante-dix sujets donnés entre 1905 et $1913^{57}$ (graphique D). Cette approche thématique permet d'individualiser nettement la médecine catholique au sein du monde médical, ce qui n'étonnera guère compte tenu du projet des fondateurs de la Société Saint-Luc. Si l'oncompare les résultats obtenus dans notre étude, aux sujets les plus abordés à cette époque dans les revues médicales généralistes ${ }^{58}$, on constate un fort intérêt pour les questions médicales appliquées aux sacrements comme le baptême ${ }^{59}$, le mariage ${ }^{60}$, de même que celles qui concernent la mort ${ }^{61}$, le jeûne et l'ascèse.

\section{Graphìne $C$ - Thènes des conférences données} ì l société s-int-Luc (18ad-1909)

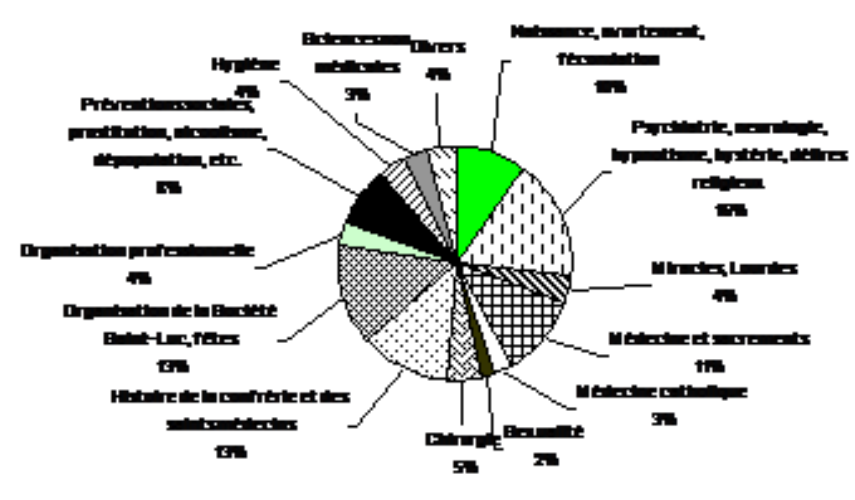

L'ensemble de ces thèmes représente plus de dix pour cent des conférences de notre corpus, ce qui dénote une première singularité. Les débats sur ces questions sacramentelles et médicales sont récurrents au sein de la Société ; en 1898, les membres du comité parisien s'interrogent sur les cas d'empêchement dans le mariage des femmes hystériques, les uns voyant dans le mariage une solution définitive à la maladie, les autres déconseillant ce qui paraît un risque pour le couple ${ }^{62}$. Les progrès de l'obstétrique (anesthésie, césarienne) et les débats précoces sur les enjeux de la fécondation artificielle interpellent évidemment les médecins de la Société Saint-Luc, 
qui comptent nombre de spécialistes dans ce domaine, et qui, en tant que catholiques, sont vigilants face à l'essor de ces problèmes que l'on ne qualifie pas encore d'éthiques.

\section{Grahique D - Thémes des confírences doumées}

ì lu Sociéfié s-int-lux (190s-1913)

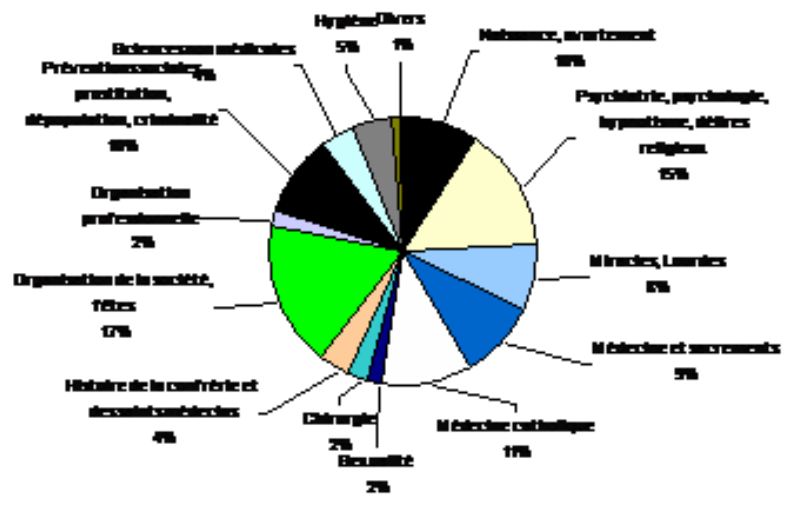

De manière transversale dans les catégories choisies, un sujet affleure, celui du danger de la perte de conscience, par l'anesthésie chimique ou hypnotique. Pour des raisons théologiques, l'idée du libre arbitre humain est fondamentale, mais en la matière, il faut se garder des conclusions hâtives qui placeraient les catholiques dans le front $\mathrm{du}$ refus de la modernité : les différends intellectuels au sein de la Société Saint-Luc sont profonds, les clivages majeurs n'étant pas forcément à rechercher dans une opposition confessionnel/laïc, mais pouvant varier selon des critères plus complexes. Derrière l'idée de la conscience pointe celle de l'inconscient psychologique ou cérébral, qui est prégnante durant ces vingt années (1884-1904), au vu des débats sur la suggestion hypnotique, qui annoncent les polémiques du temps de la fondation psychanalytique ${ }^{63}$. L'ensemble des contributions gravitant autour de la nature de l'âme ou du cerveau, des formes de suggestions et de leur puissance thérapeutique, des pathologies nerveuses ou psychologiques, constituent sans conteste le premier champ de recherche et d'exposition des médecins catholiques (seize pour cent). En octobre 1888, le Docteur Ferrand énonce ainsi le programme idéal de la Société :

"Questions de physiologie générale, de psychologie physiologique, d'influences pathogéniques d'ordre moral ou immoral, hygiène supérieure par conséquent, traitement des affections psychiques et moyens moraux d'action thérapeutique, toutes les conditions ou la déontologie se trouve aux prises avec l'intérêt ou avec la convenance ; questions d'anthropologie et d'exégèse même, il en est beaucoup qui nous touchent et que nous devons étudier, d'autant plus que les autres affectent davantage de la négliger, ou bien de les résoudre d'une façon contraire à ce que commandent nos sentiments et nos principes. Tel doit être l'objet de nos travaux. Et cet objet s'impose surtout aujourd'hui que la lutte religieuse est descendue sur ce terrain et que l'apologétique scientifique est plus que jamais à l'ordre du jour $»{ }^{64}$. 
31 Si l'on ajoute à ces thèmes médico-psychologiques les conférences ayant trait aux miracles de Lourdes, abordés le plus souvent sous l'angle de la suggestion, un sujet sur cinq relève de questions psychiques. Les médecins catholiques sont donc sur un terrain de prédilection lorsqu'ils abordent ce domaine médical des relations du corps et de l'esprit, des thérapies morales et psychologiques. Ce terrain d'étude, encore largement en friche à la fin du XIX ${ }^{e}$ siècle et parfois considéré comme marginal par les autres médecins, révèle que l'opposition à la modernité est loin d'être unanime dans la Société Saint-Luc. À l'inverse, les sujets touchant à l'hygiène et aux questions sociales (prostitution, enfance délinquante, alcoolisme, dépopulation) sont minorés alors qu'ils sont prépondérants dans les organes médicaux imprégnés des conceptions hygiénistes, diffusant au sein du corps social une conception nouvelle du corps et de l'espace ${ }^{65}$. La médecine catholique est une médecine religieuse et sociale, mais le champ de son action ne couvre pas l'ensemble des préoccupations sociales du moment. Cette répartition des thèmes traités dans les conférences de la Société évolue assez peu dans les années 1905-1913 (Graphique D), si l'on excepte la part plus grande donnée à la défense du site de Lourdes et de ses vertus thérapeutiques, menacées par les hygiénistes, défense amplifiée par les polémiques scientifiques et politiques autour du vote de la loi de séparation de l'Église et de l'État. On peut aussi noter la multiplication des textes qui tentent une définition du statut et des missions du médecin chrétien dans la société et dans l'Église, ainsi qu'un accroissement des études épidémiologiques concernant les maladies contagieuses, syphilis et tuberculose en tête. À l'intérieur des catégories thématiques on perçoit des évolutions plus fines; la question de l'avortement prime sur celle de l'embryologie, la psychologie remplace la neurologie, le débat sur la mystique s'essouffle, la jeunesse criminelle préoccupe plus que l'alcoolisme. Plus surprenant, alors que la question de la natalité devient cruciale au tournant du siècle puisque la France n'assure plus le renouvellement des générations depuis 1890 , la question de la dépopulation et le combat contre les idées malthusiennes ne sont guère en débat chez les médecins catholiques avant 1914. Comme Martine Sévegrand a pu le montrer, la politique nataliste est patriote avant d'être catholique ${ }^{66}$. Un exemple d'apologétique médicale : la défense du sanctuaire de Lourdes

L'histoire des apparitions et du pèlerinage de Lourdes s'est enrichie depuis quelques années de développements sur les guérisons, sur les thérapeutiques mises en œuvre près de la grotte de Massabielle, sur les débats et les polémiques qui agitèrent le monde médical et au-delà l'ensemble de la société à travers la presse à grand tirage ${ }^{67}$. Le rôle des médecins, et notamment des catholiques, dans les dernières décennies du XIX ${ }^{\mathrm{e}}$ siècle, apparaît de plus en plus important dans le processus de vérification des pathologies et des guérisons miraculeuses, vérification nécessaire à la préservation de l'aura du sanctuaire. La création du Bureau des constatations médicales de Lourdes a été évoquée de façon contradictoire par quelques auteurs ${ }^{68}$ : qui de l'Église ou des médecins a donné l'impulsion de départ? La Société Saint-Luc joue-t-elle un rôle dans cette création?

Les autorités diocésaines, comme toujours dans le cas d'apparitions, forment une commission dans laquelle siègent des médecins chargés d'informer les ecclésiastiques, qui jugent en dernier ressort de la qualité des guérisons. Dans les années 1870, devant l'essor du premier site de pèlerinage de France, voire du monde catholique, face à la multiplication des guérisons présentées comme miraculeuses, s'impose l'idée d'une organisation médicale permanente. La création du Bureau des Constatations en 1884 est 
exactement contemporaine de celle de la Société Saint-Luc et les quatre premiers présidents du bureau, nommés par l'évêque de Tarbes, ont été choisis parmi les adhérents de la Société. Il s'agit de Dunot de Saint-Maclou, président de 1884 à 1891, de Gustave Boissarie de 1891 à 1917, du docteur Cox (intérimaire) de 1917 à 1920 et de Edouard Le Bec, de 1920 à $1923^{69}$. D'autres membres de la Société font partie du Bureau ou font des séjours auprès de leurs confrères, attirés comme nombre de médecins, pas exclusivement catholiques, par la réputation croissante des lieux ${ }^{70}$. L'ouverture affichée de cet organisme de contrôle, qui permet à tous les médecins de collaborer aux examens, est en fait limitée par cette prépondérance des médecins catholiques au sein de la direction permanente. Le Bureau des constatations reste donc largement dominé par les catholiques, ce qui pourtant ne freine pas la restriction croissante des approbations scientifiques envers les guérisons miraculeuses ${ }^{71}$.

Dès 1884 se fait sentir le besoin d'une collaboration médicale étendue afin de rédiger les certificats de guérison, rôle dévolu aux médecins du Bureau, mais aussi les certificats médicaux qui établissent les pathologies de départ, tâche qui s'avère beaucoup plus délicate à mettre en place ${ }^{72}$. Les médecins de la Société Saint-Luc tentent, semble-t-il avec quelques difficultés, de répondre à ce besoin, à la demande des assomptionnistes ${ }^{73}$. Cette collaboration s'amplifie après 1900 , sous l'impulsion de Camille Féron-Vrau, dont l'objectif est de renforcer la clinique de Lourdes et de développer l'accueil des professionnels. Peut-être faut-il voir dans l'essor du comité du Sud-Ouest un effet du pèlerinage et des nouvelles fonctions dévolues aux médecins ${ }^{74}$. Lorsqu'en 1906, les piscines du sanctuaire sont menacées de fermeture par les hygiénistes, la Société Saint-Luc se place à la tête d'une campagne de résistance dans les milieux médicaux sous la forme d'un référendum ${ }^{75}$. Henri Dauchez, lance un plan de conférences sur les miracles médicaux de Lourdes en direction des milieux populaires, et en novembre 1910, le docteur Van der Elst ${ }^{76} \mathrm{~s}^{\prime}$ attache à porter la contradiction dans les milieux ouvriers sur une des questions les plus brûlantes du moment. Face à la remise en cause des attaches populaires du catholicisme (le rôle des saints dans les guérisons miraculeuses ou la croyance dans les démons possesseurs notamment ${ }^{77}$ ), la réponse catholique allie rénovation théologique et appel à la science médicale. La bataille apologétique ne se fait pas tant sur le plan de l'hygiène que sur celui des phénomènes de suggestion et de psychologie des foules, clé de voûte de la critique anticléricale.

Cette défense unanime du sanctuaire par les médecins catholiques masque en fait un débat interne qui prend parfois un tour polémique. Si les publications du bulletin de la Société en témoignent, elles atténuent cependant la portée des échanges conflictuels perceptibles au sein de la Société Saint-Luc. Après la parution de l'ouvrage de Gustave Boissarie, Les Grandes guérisons de Lourdes, en 1900, ses confrères catholiques mettent en discussion les méthodes du récent Bureau des constatations. Le docteur René Le Fur, membre du comité de Paris, est le plus sévère et sa critique porte en premier lieu sur l'insuffisante méfiance envers les pathologies nerveuses :

«Les cas de guérisons miraculeuses d'ulcères de l'estomac rapportés par le docteur Boissarie peuvent être, à notre avis, interprétés d'une autre façon. On sait le rôle que jouent souvent l'élément nerveux et l'hystérie dans la pathogénie de l'ulcère simple de l'estomac [...] Quant aux maladies purement nerveuses (hystéries, épilepsie, paralysies, aphonies), bien que le docteur Boissarie voie souvent dans leur guérison une influence surnaturelle, nous croyons qu'elles ne peuvent en rien servir la cause du miracle, 
qu'elles ne peuvent au contraire que lui nuire. [...] Le médecin catholique doit, en effet, se montrer encore plus exigeant que le médecin indifférent quand il s'agit d'affirmer le miracle. [...] Il y a un retour mystérieux et incontestable vers le surnaturel et les médecins n'auront pas peu contribué à ce mouvement de salut " ${ }^{78}$.

La critique interne ne se fait pas au détriment de la notion de miracle, mais vise à épurer le sanctuaire des fausses guérisons, des cas nerveux ou psychologiques, afin de préserver le mystère et la puissance de Lourdes. La science médicale devient le vecteur du surnaturel et non son fossoyeur. D'autre part, l'auteur n'épargne pas l'organisation du pèlerinage, l'autorité des directrices de salle et le rôle des brancardiers, qui dépassent souvent les prérogatives des médecins ${ }^{79}$. Cette attitude, qui peut sembler contredire nombre d'écrits catholiques contemporains, est en fait répandue chez les scientifiques croyants. En effet, le comité parisien partage largement l'avis du docteur Le Fur, mais décide de tempérer les critiques publiées afin de ne pas nuire à la bonne réputation dont jouit le docteur Boissarie dans le monde catholique ${ }^{80}$.

À cette époque ces médecins prennent ainsi dans le monde catholique une place fondamentale, et du fait de l'écho donné à ces questions dans la presse, dans les conversations quotidiennes, dans les romans ${ }^{81}$, dans les conférences publiques spectaculaires ${ }^{82}$, dans le monde scientifique et dans la société entière. Cela au moment même où les médecins agnostiques ou athées, voire anticléricaux, revisitent le patrimoine de l'Église, ses figures, ses pratiques, afin de comprendre la puissance thaumaturgique du religieux, et ainsi d'en laïciser les gestes ${ }^{83}$. Cette évolution tendait à marginaliser les prêtres dans les hôpitaux, dans les sanctuaires, mais aussi dans l'imagerie de la maladie et de la guérison, dans ses représentations populaires courantes. L'action des docteurs Boissarie, Le Bec, Dauchez, Cox et des membres de la Société Saint-Luc s'inscrit donc dans une perspective de construction d'une apologétique scientifique médicale, qui est aussi significative dans la défense des personnels congréganistes hospitaliers, dans la critique des pratiques hypnotiques, dans la réhabilitation des états mystiques, dans la campagne contre la crémation des corps.

Les mutations de la Société Saint-Luc : internationalisation et crise de croissance (1904-1905)

Les années 1904 et 1905 marquent une étape dans l'évolution de la Société Saint-Luc. Celle-ci réorganise ses structures, suite au changement de direction, qui entérine la marginalisation des érudits et des savants reconnus au bénéfice des apologètes et des militants, tels que Henri Dauchez, Octave Pasteau et Edouard Le Bec. L'effort sur le recrutement porte ses fruits ${ }^{84}$. Tandis que des voix s'élevaient à la fin du siècle pour tempérer l'image par trop dévote du groupement médical, cause présumée de la stagnation des effectifs, on assiste à un renversement complet, puisque le renforcement quantitatif de la Société apparaît lorsqu'elle s'investit le plus dans la défense de Lourdes ou auprès des autorités romaines. Le contexte n'est plus le même, il est vrai, entre les années de la marginalité relative (1880-1890) et celle de l'insertion scientifique et sociale (1900-1910). Le climat intellectuel, culturel, littéraire et scientifique s'est modifié depuis la fin du XIX siècle, les polémiques se sont apaisées, et pour un praticien, entrer dans l'organisation des médecins catholiques n'entraîne sûrement pas, en 1910, railleries ou représailles. Le caractère confessionnel de la Société devient positif alors qu'il était un frein auparavant. L'effet mécanique de la 
croissance des effectifs médicaux français de même que les liens étroits tissés avec les étudiants des Conférences Laënnec expliquent également ce rebond des effectifs.

Graphique E - Évolution des effectifs par régions entre 1888 et 1908

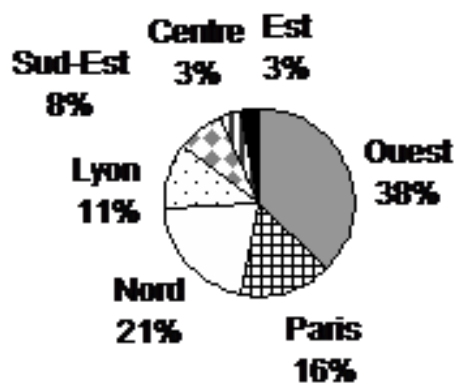

18:8:8

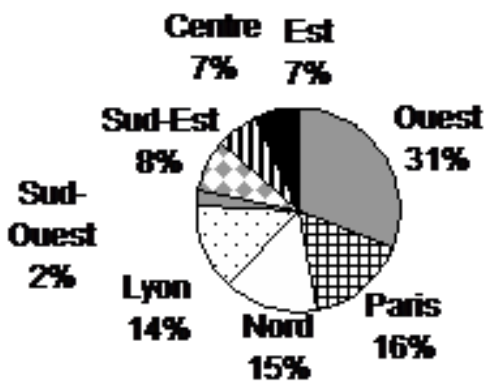

1896

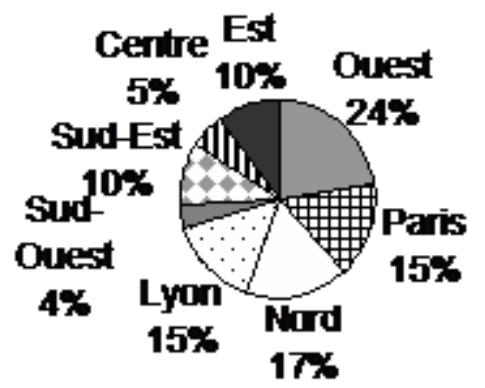

1908

L'analyse de l'évolution régionale des effectifs de la Société Saint-Luc sur le long terme (graphique E) montre le recul relatif et la stagnation en nombre d'adhérents des comités de l'Ouest (Nantes, Rennes, Rouen, Laval, Le Mans), ainsi que la baisse relative récente $\mathrm{du}$ Nord et $\mathrm{du}$ Centre, régions de forte implantation catholique. Les interprétations de ce fléchissement du recrutement peuvent être multiples, mais il est probable qu'il est la conséquence d'une mutation de la société ${ }^{85}$.

41 On distingue en effet deux grandes évolutions qui peuvent paraître contradictoires : d'une part un resserrement des liens avec l'Église et avec Rome, entre 1899 et 1904 ; d'autre part une ouverture sur la société, sur les autres groupements professionnels 
médicaux, surtout à partir de 1905. Dans un premier temps les relations avec les institutions catholiques deviennent plus étroites: les médecins appuient la vocation sociale de l'Église en créant par exemple des dispensaires, tandis que l'Église de son côté favorise l'épanouissement professionnel de quelques-uns en créant des postes de médecin inspecteur des écoles libres ${ }^{86}$. Ce rapprochement est notable non seulement lors de la campagne de défense du sanctuaire de Lourdes, mais surtout lors de l'organisation du pèlerinage des médecins à Rome en avril 1904. Camille Féron-Vrau, Gustave Boissarie et Henri Dauchez, qui sont à l'origine de ce projet, se sont aussi distingués dans la lutte contre les hygiénistes et dans l'extension du recrutement. Les liens entre Lourdes, le pèlerinage romain et l'essor de la Société sont d'ailleurs soulignés par l'intitulé suivant: "Pèlerinage des médecins catholiques à Rome en l'honneur de Notre-Dame de Lourdes, à l'occasion du cinquantenaire de la proclamation du dogme de l'Immaculée Conception ». L'acte collectif placé sous le signe de la dévotion mariale est une reconnaissance de l'infaillibilité pontificale associée à la proclamation du nouveau dogme de l'Église dans la seconde moitié du XIX ${ }^{e}$ siècle. La réception à Rome témoigne de la sanction du nouveau rôle du médecin, qui est témoin du miracle en même temps qu'il en est le défenseur par sa fonction apologétique ${ }^{87}$. Pour Rome, la réception officielle du corps médical français, dont l'imagerie véhicule une caricature anticléricale, est un geste fort de Pie X, dans la lignée de la politique de science catholique développée par Léon XIII, dont le pontificat s'est achevé un an plus tôt. Le convoi ferroviaire, symboliquement ouvert par les miraculés de Lourdes choisis par les médecins, montre que la Société réussit à fédérer autour de son action les familles des malades, les étudiants catholiques ainsi que la Faculté libre de médecine de Lille.

Le moment du pèlerinage est aussi l'occasion de contacts avec les sociétés médicales catholiques européennes, la Société Saint-Luc jouant depuis plusieurs années un rôle dynamique dans la création d'un réseau international de sociétés fondées sur les mêmes principes et les mêmes statuts. L'internationalisation s'opère par l'adhésion de membres étrangers à la Société et par la participation à la formation de sociétés similaires à la société française en Belgique, en Espagne, en Italie, aux Pays-Bas et en Angleterre à partir de $1904^{88}$. La société française, qui est la plus ancienne, sert de modèle dans un réseau encore embryonnaire qui est centralisé à Rome. Ces développements internationaux de la médecine catholique s'étendent dans les années vingt ${ }^{89}$.

43 L'affirmation du caractère confessionnel de la Société, le resserrement des liens avec les catholiques européens et avec Rome n'empêchent pas une ouverture plus grande hors de la sphère de la médecine catholique, comme l'illustre le discours d'Eugène Vincent le 8 octobre 1905, intitulé "Sur l'orientation nouvelle à donner aux études et aux énergies des sociétés Saint-Luc: nécessité d'entrer dans le mouvement social moderne ». Dans une République qui s'apprête à voter les lois de séparation de l'Église et de l'État (9 décembre 1905), dans une société qui voit se multiplier les syndicats professionnels, notamment chrétiens, tandis que le Sillon et l'Action Française prônent l'engagement social et politique des catholiques, Eugène Vincent, qui est alors viceprésident de la Société Saint-Luc et président du comité lyonnais, toujours influent, propose une orientation nouvelle pour l'action des médecins catholiques. Le modèle choisi n'est pas celui des grandes sociétés savantes, ni celui des syndicats, mais plutôt, 
ce qui surprendra, celui de la Franc-maçonnerie, le double objectif avoué étant de renforcer la solidarité interne et de développer les contacts extérieurs :

«La démocratie aux tendances socialistes est un océan orageux sur lequel on nous a lancés et sur lequel il faut nous résigner à diriger nos vaisseaux, si nous ne sommes pas résignés à périr. Regrettons le passé, tant que vous voudrez; mais manœuvrons quand même sur les flots tumultueux tels qu'ils sont surgis des entrailles du sol moderne. Volontiers je vous dirais : courage, jetez-vous dans la bataille de la vie du $\mathrm{XX}^{\mathrm{e}}$ siècle, telle que la révolution l'a faite et prenez votre rang d'honneur dans la lutte active qu'elle impose à tous les citoyens ${ }^{90}$.

Le thème de la défense catholique s'estompe face à celui de l'intervention sociale, dont les médecins esquissent le programme: assurances collectives, hygiène. Cette orientation ouverte et active de la Société n'est pas partagée par tous les membres et des tensions fortes apparaissent lorsqu'il s'agit de discuter la transformation de l'organisation selon les statuts de la loi de 1901 ou selon un modèle syndical. Il existe aussi des tentatives de rapprochement avec d'autres organisations chrétiennes qui ont pour but d'obtenir des avantages matériels, ou de capter la clientèle catholique ${ }^{91}$. Dans ce débat qui court sur plusieurs années, la dénomination catholique de la Société est parfois mise sur la sellette, avant d'être définitivement conservée ${ }^{92}$.

Des questions posées par l'histoire de la médecine catholique

On peut donc parler d'une " médecine catholique ", singulière quant à ses thèmes de prédilection, fortement liée à l'Église et à Rome, construite en opposition aux attaques scientifiques anticléricales et a-religieuses, et exercée dans une optique apologétique qui place à l'arrière-plan, au moins dans un premier temps, la recherche scientifique et l'action professionnelle. Dans cette première phase de leur histoire, les hommes de la Société Saint-Luc unissent deux désirs : pratiquer la médecine en tant que catholiques dans un milieu a priori hostile, favoriser l'éclosion d'une science originale fondée sur une morale et une doctrine, à des fins de défense ou de conversion. L'expression " médecine catholique ", toujours revendiquée par les membres de la Société, est donc justifiée. Mais elle trouve néanmoins ses limites dans le cadre sociologique, car les médecins catholiques s'inscrivent dans un groupe social qui transcende les appartenances confessionnelles. D'autre part, leur milieu intellectuel subit des évolutions qui régissent les pratiques scientifiques et médicales au-delà des affinités culturelles, religieuses ou politiques. En 1903, la notion de médecine catholique est encore en débat au sein de la Société Saint-Luc et oppose deux conceptions, celle de Xavier Gouraud: " la science n'est pas catholique, elle est la science. Il y a sans doute des savants catholiques mais lorsqu'on est médecin catholique cela ne veut pas dire qu'on fasse de la médecine catholique » et celle d'Edouard Le Bec, pour qui « il peut y avoir une manière chrétienne de faire de la médecine ${ }^{93}$. La multiplication des manuels de médecine chrétienne entre 1900 et 1940 montrent que les débats se poursuivent et que cet idéal se concrétise auprès des fidèles ${ }^{94}$.

47 À la confluence de l'histoire de la médecine et de l'histoire du christianisme, de l'histoire de la science et de l'histoire religieuse, de l'histoire sociale et de l'histoire des croyances, l'histoire de la médecine catholique, qu'il faudrait étendre à la médecine chrétienne, permet d'apporter une contribution aux voies récemment explorées et d'ouvrir quelques champs de recherches. Les bilans récents de l'histoire de la médecine ont mis l'accent sur les nuances apportées au tableau d'une sécularisation rapide des pratiques thérapeutiques, principalement autour du rôle des congréganistes dans la 
modernisation des services hospitaliers : la formation d'un cadre institutionnel médical laïc, la déchristianisation partielle des populations n'impliquent pas automatiquement la disparition de pratiques thérapeutiques imitées ou reprises partiellement ou intégralement des pratiques religieuses ${ }^{95}$. Le dépassement du discours politique fondé sur l'antagonisme entre science et religion doit être privilégié dans l'étude de ces pratiques ${ }^{96}$. Les institutions médicales chrétiennes, hôpitaux et asiles d'aliénés par exemple, témoignent de confrontations significatives qui donnent à voir les conflits, les synthèses, les accommodations, susceptibles de régler les relations entre deux approches opposées dans les discours. L'extension chronologique de cette étude en amont et en aval montrerait la complexité de ces relations, qu'une focalisation sur la période d'exacerbation des tensions sociales et politiques à la fin du XIX ${ }^{\mathrm{e}}$ siècle, tend à atténuer. Comme Michel Lagrée a pu le montrer dans le domaine des techniques, il est souvent nécessaire de revisiter les clivages schématiques opposant modernité et tradition religieuse dans la sphère technique et scientifique, à la façon dont l'opposition parfois commode entre culture populaire et culture des élites a été partiellement révisée ${ }^{97}$. Les débats au sein de la Société Saint-Luc, les usages thérapeutiques différents qui ont cours dans les institutions chrétiennes, incitent à inventer d'autres lignes de clivages, distincts de l'appartenance confessionnelle.

La médecine chrétienne, lorsqu'elle existe en tant que telle, peut aussi être considérée comme faisant partie des médecines marginales, marginalisées, comme un recours en cas d'échec de la médecine officielle, mais aussi comme une branche originale de la médecine dans les domaines touchant à la vie religieuse, à la psychologie, ou dans ceux qui intéressent directement la vie de l'Église: discernement physiologique et psychologique des miracles, des possessions, des états mystiques, recrutement des séminaristes, etc. L'esquisse d'une science catholique à la fin du XIX ${ }^{\mathrm{e}}$ siècle aboutit dans l'entre-deux-guerres au développement des réseaux et des publications ouvertement consacrés à la diffusion d'une médecine catholique dans les cercles des fidèles, les ecclésiastiques participant plus qu'auparavant à des réseaux qui deviennent médicoreligieux ${ }^{98}$. La Société Saint-Luc, connaissant un fort essor dans ces années, participe à ces nouvelles formes de collaboration qui donnent naissance à une rénovation des pratiques dans la seconde moitié du XXe siècle.

On peut aussi s'interroger sur le rôle du médecin chrétien dans le processus lent de sécularisation de la société. Figure contradictoire de la fin de siècle, le médecin chrétien porte quelques thèmes fondamentaux de la réflexion sociale du $\mathrm{XX}^{\mathrm{e}}$ siècle, ceux de la naissance, de la mort, et au croisement celui de l'eugénisme, ceux de la libre conscience et de la souffrance psychique. La figure du médecin est-elle totalement étrangère à celle du prêtre, à son pouvoir de guérison par la parole, à sa capacité à pénétrer l'intimité familiale, à sa soumission au secret ? François Laplantine souligne la " ferveur structurellement religieuse des médecins pour la médecine " ${ }^{99}$. Le prêtre, en retour, emprunte-t-il à la science médicale quelques nouveaux savoirs, appliqués dans les gestes quotidiens? La singularité du médecin chrétien est de se trouver au croisement de ces deux figures symboliques. Ce point de contact devient particulièrement probant si on focalise son attention sur le domaine psychologique, psychiatrique et psychanalytique. L'histoire médicale, l'histoire de la psychologie comme l'histoire religieuse peuvent ainsi s'enrichir mutuellement. 


\section{NOTES}

1.. Texte cité dans Bulletin Médical de la Société Saint-Luc, Saint-Côme et Saint-Damien, novembre 1904, p. 213. Sur le Cardinal Mercier, R. Aubert, Le Cardinal Mercier (1851-1926), un prélat d'avant-garde, Académia, Presses Universitaires Louvain, 1994.

2. Michel LAGRÉE et François LEBRUN [dir.], Pour l'histoire de la médecine. Autour de l'œuvre de Jacques Léonard, Presses Universitaires de Rennes, 1994 ; Revue d'Histoire Moderne et Contemporaine, « Médicalisation et professions de santé XIX ${ }^{\mathrm{e}}-\mathrm{XX}^{\mathrm{ee}}$ », numéro spécial, octobre-décembre 1996. Une synthèse bibliographique est publiée dans Annales Histoire, Sciences sociales, février 2001. Pour le bilan historiographique de l'histoire médicale récente, voir Olivier FAURE, « Les Voies multiples de la médicalisation », dans Revue d'histoire moderne et contemporaine, octobre 1996, pp. 574-577. Une approche européenne dans Mary LINDEMANN, Medicine and society in early modern Europe, Cambridge New York, University Press, 1999.

3.. Pierre GUILLAUME, Médecins, Église et foi, XIX ${ }^{e}-X X^{e}$ siècles, Paris, Aubier, 1990, pp. 90-94 et pp. 107-112 ; J. LIEFOOGHE, « Médecine et pensée chrétienne en France au XX siècle », dans Mélanges de sciences religieuses, tome 53, juin 1996, pp. 119-144 ; François LEBRUN, «L'Église face à la maladie et à la guérison entre Trente et Vatican II ", dans Jacques MAITRE ET Françoise LAUTMAN [dir.], Gestions religieuses de la santé, Colloque de l'Association Française de sociologie religieuse, mars 1993, Paris, L'Harmattan, 1995, pp. 15-23 ; M. C. PITASSI, « Le monde catholique face aux sciences au XVII ${ }^{\mathrm{e}}$ et XVIII ${ }^{\mathrm{e}}$ siècles ", dans Olivier FATIO, Les Églises face aux sciences, du Moyen-Age au XX ${ }^{e}$ siècle, Paris, Droz, 1991, pp. 91-108. Sur un aspect plus précis, Yves Marie BERCÉ, « Le Clergé et la diffusion de la vaccination ", dans Revue d'Histoire de l'Église de France, janv. 1983, pp. 87-106. Sur les rapports entre science et religion voir Claude LANGLOIS, "Science et religion ", Christianisme et science, Paris, A.F.H.R.C., 1989, p. 217 ; Georges MINOIS, L'Église et la science, 2 vol., Paris, Fayard, 1991. Des travaux récents ont apporté certains éclairages sur l'action des médecins catholiques lors du pèlerinage de Lourdes ou dans le domaine de la procréation : Ruth HARRIS, Lourdes. La grande histoire des apparitions, des pèlerinages et des guérisons, Paris, J-C. Lattès, 2001; Martine SEVEGRAND, Les Enfants du bon Dieu. Les Catholiques français et la procréation au XXe siècle, Paris, Albin Michel, 1995 ; Anne CAROL, Histoire de l'eugénisme en France. Les médecins et la procréation, XIX ${ }^{e}-X X^{e}$ siècles, Paris, Éditions du Seuil, 1995, pp. 262-268.

4.. Si l'on excepte les travaux de Claude Langlois sur la vulgarisation médicale qui évoquent l'œuvre du Père J. Debreyne, les médecins chrétiens du XIX siècle n'ont été l'objet ni d'études collectives, ni d'études biographiques. On peut citer, pour les débuts $\mathrm{du} \mathrm{XX}^{\mathrm{e}}$ siècle, l'ouvrage collectif, sous la direction de Régis LADOUS, Médecine humaine, médecine sociale. Le Docteur René Biot et ses amis (1889-1966), Paris, Éditions du Cerf, 1992. Sur l'anticléricalisme des médecins, on peut se référer à l'exemple des psychiatres, Ijan GOLDSTEIN, Consoler et classifier. L'essor de la psychiatrie française, Paris, Les Empêcheurs de penser en rond, Institut Synthelabo, 1997, et aux exemples donnés par Jacqueline LALOUETTE, La Libre pensée en France (1848-1940), Paris, Albin Michel, 1997. La question de la sécularisation relative de la société est posée dans les deux historiographies médicale et religieuse. René RÉMOND, Religion et société en Europe. La Sécularisation aux XIX ${ }^{e}$ et XX $X^{e}$ siècles (1780-2000), Paris, Éditions du Seuil, 2001 ; Philippe BOUTRY, « L'Église et la civilisation moderne de Pie IX à Pie X », dans Le Deuxième concile de Vatican 
(1859-1965), Ecole française de Rome, 1989 ; Émile POULAT, « Catholicisme et modernité ", dans Modernistica. Horizons, physionomies, débats, 1982, p. 79 ; Étienne FOUILLOUX, «Intransigeance catholique et monde moderne, XIX ${ }^{\mathrm{e}}-\mathrm{XX}^{\mathrm{e}}$ siècles », dans Revue d'Histoire ecclésiastique, $\mathrm{n}^{\circ}$ 96, janvier 2001, pp. 71-86 ; Régis LADOUS, « Le Magistère catholique au défi de la modernité ou l'impossible distinction des sciences (1870-1920) », dans Revue d'Histoire ecclésiastique, juillet 2000, p. 651.

5.. Jacques LÉONARD, La Médecine entre les pouvoirs et les savoirs : histoire intellectuelle et politique de la médecine française au XIXe siècle, Paris, Aubier, 1981 ; Olivier FAURE, Les Français et leur médecine au XIX ${ }^{e}$ siècle, Paris, Belin, 1993.

6.. L'expression est mennaisienne et date des années 1830 . Voir sur la genèse de ce concept dans François LAPLANCHE, « La Notion de science catholique : ses origines au début du XIX ${ }^{e}$ siècle ", dans Revue d'Histoire de l'Église de France, 1988, pp. 63-90; du même auteur, Dictionnaire du monde religieux dans la France contemporaine, volume 9, Les Sciences religieuses 1800-1914, Paris, Beauschène, 1996.

7.. François LAPLANCHE et Claude LANGLOIS, La Science catholique : L'Encyclopédie de Migne (1844-1873) entre apologétique et vulgarisation, Paris, Sciences en Situation, 1992. Parmi les volumes de l'Encyclopédie ayant trait à la médecine, voir le Dictionnaire de physiologie ( $58^{\mathrm{e}}$ volume de la $3^{\mathrm{e}}$ série publié en 1861 ). Pour les congrès scientifiques catholiques de la fin du XIXe siècle, Francesco BERETTA, Mgr d'Hulst et la science chrétienne. Portrait d'un intellectuel, Paris, Beauschène, 1996.

8.. Peu après l'interruption de ses études parisiennes qui l'ont conduit à fréquenter le célèbre professeur, il est d'abord médecin des épidémies, au temps du choléra, en 1850, puis chirurgien de l'hôpital civil du Mans en 1870. Très éclectique comme auteur, il publie une étude locale de sciences naturelles, un traité d'hygiène pratique, un opuscule sur le Rosaire et la dévotion au Sacré-Cœur.

9.. L'autre moine de Solesmes est médecin, c'est le Dr Fournier. Les autres membres fondateurs de la Société sont les docteurs Le Bêle et Tournet-Desplantes (Le Mans), Regnault et Raymond-Petit (Rennes), Lelièvre (Séez), Renier (Angers), Ferrand et Dauchez (Paris), De La Mardière (Poitiers), Vintrebert (Lille), Coutenot (Besançon), Seux et Audibert (Marseille), Delore (Lyon). La forte représentation des médecins de l'ouest est due non seulement à la localisation de la réunion (Le Mans) mais aussi à la géographie du monde catholique et des facultés libres. L'évêque du Mans, qui a provoqué la réunion avec le Docteur Le Bêle, est agonisant ; Dom Couturier, abbé de Solesmes, est donc délégué à sa place, probablement en raison de la proximité de l'abbaye. Cette présence fortuite des Bénédictins inaugure la présence de prêtres médecins de l'abbaye dans la Société, dont le plus réputé est Dom Sauton.

10.. Sur le syndicalisme médical, voir Bénédicte VERGEZ-CHAIGNON, « Le syndicalisme médical français de sa naissance à sa refondation ", dans Revue d'histoire moderne et contemporaine, octobre-décembre 1996, pp. 709-734 et Pierre GUILLAUME, Le Rôle social du médecin depuis deux siècles, Comité d'histoire de la. Sécurité sociale, 1996, pp. 117-142. 11.. Dom Couturier, Discours de fondation de la Société Saint-Luc, 27 septembre 1884, reproduit dans Bulletin médical de la Société Saint-Luc, Saint-Côme et Saint-Damien, janvier 1927, p. 8 (pour les pages suivantes : Bull. Méd. Soc. S. L.).

12.. Au petit groupe de médecins qui le sollicite, Henri Lacordaire répond : « Vous me consultez sur l'établissement d'une société de médecins au point de vue de la conservation et de la propagation de la foi catholique. Je suis persuadé, Monsieur, qu'une telle association, fondée sur un but clair et des moyens précis et par un petit nombre d'hommes dévoués ferait un très grand bien. Il est impossible de rien produire 
de fort, d'étendu et de durable, en dehors de la forme sociale. C'est pourquoi JésusChrist a établi l'Église et ne s'est pas contenté de donner sa parole et son sang au monde. L'esprit d'union ou plutôt d'unité est l'esprit de toutes les œuvres chrétiennes. Il n'en est pas moins difficile de réaliser cet esprit, ou si vous aimez mieux de créer un corps chrétien agissant dans un but chrétien, par des moyens du même ordre. Aujourd'hui surtout qu'il y a si peu d'accord, même entre des Catholiques, il est plus que jamais mal aisé de fonder une association, précisément parce que cela est plus nécessaire que jamais. Si vous me permettez de vous dire ma pensée, je crois qu'un élément de succès, en pareille matière, est de commencer avec un très petit nombre d'hommes, déjà unis par ces rapprochements que ménage la Providence, et de poser le but et les moyens de l'association d'une manière très déterminée. Par exemple notre but sera-t-il pratique ou scientifique, ou tous les deux ensemble ? En tant que pratique, des médecins chrétiens unis entre eux peuvent faire une foule de bonnes et saintes choses, visiter gratuitement des malades, porter ceux qu'ils visitent à la pratique des sacrements de l'Église, les avertir du danger, recueillir tous les faits qui intéressent la foi dans leur ordre d'action, etc. Le but scientifique serait de se défendre, soi et les autres, des préjugés de la médecine matérialiste, de poser les bases d'une nouvelle école spiritualiste, d'avoir des discussions en ce sens, de propager les bons livres médicaux, etc. [...] En résumé, Monsieur, je souhaite passionnément la réalisation de votre excellente pensée et je suis persuadé qu'elle réussira d'autant plus que vous vous éloignerez davantage de la forme académique pour revêtir la forme catholique », Lettre du Père Henri Lacordaire (Rome) au docteur Tessier, 12 mai 1840, reproduite dans Henri DAUCHEZ, «Tentative de restauration de la Société de Saint-Côme, de Saint-Luc et de Saint-Damien en 1840 ", dans Bull. Méd. Soc. S. L., mai 1898, p. 62. Ce groupe de médecins ne prend guère $\mathrm{d}$ 'ampleur et se fond très rapidement au sein de l'ordre de Saint-Dominique, restauré en 1843.

13.. Statuts de la Société Saint-Luc, Titre II, art. 4 et 5, Assemblée générale, oct. 1888, p. 4, Archives C.C.M.F.

14.. Bull. Med. Soc. S. L., juin 1922, pp. 175-177.

15.. Dr FERRAND, Assemblée générale de la Société Saint-Luc, 17 oct. 1886, p. 24, Archives C.C.M.F.

16.. Citons parmi ces pèlerinages locaux, Luzarches bien sûr mais aussi Sainte-Anned'Auray, qui paraît le plus fréquenté.

17.. Société Médicale de Saint-Luc, Saint-Côme et Saint-Damien, Assemblée générale du 19 oct. 1890, Paris, 1891, p. 15, Arch. C.C.M.F (Archives du Centre catholique des médecins français).

18.. Les accusations médicales portent sur la psychologie des états mystiques et des états démoniaques. Sur la mise en cause de la possession et de l'exorcisme, Hervé GUILLEMAIN, «Démons ou déments ? L'exorcisme face aux sciences psychiques, XIX ${ }^{e_{-}}$ XX siècles ", dans Revue d'histoire de l'Église de France, juil. 2001, pp. 439-471.

19.. Sur l'Encyclique Humanum Genus et sa critique de la sécularisation, voir Philippe LEVILLAIN [dir.], Dictionnaire historique de la papauté, Paris, Fayard, 1994.

20.. Le pèlerinage de Luzarches subsiste au XIX $X^{\mathrm{e}}$ siècle, au moins jusqu'en 1864, et est fréquenté par des médecins réputés, Maisonneuve, Cruveilhier et probablement Laënnec. Sur ces points, voir Bull. Méd. Soc. S. L., janv. 1927, pp. 3-7. Si quelques évêques font partie des bienfaiteurs de la Société (archevêque d'Aix à Lyon, Mgr Richard et Mgr d'Hulst à Paris) et participent parfois aux réunions, en réalité l'œuvre est le fait de médecins laïcs. La présence des prêtres est faible et leur influence dans les comités 
directeurs est négligeable, si l'on excepte quelques prêtres médecins, qui n'ont cependant aucun rôle dans l'organisation. Le comité du Centre, qui n'est qu'embryonnaire en 1895 , se sert des Semaines religieuses et mobilise les diocèses afin d'approcher l'ensemble des médecins : trente-cinq prêtres servent la cause de la Société dans le Centre de la France ; cette expérience de collaboration dans le recrutement semble unique.

21.. DOM COUTURIER, Discours de fondation de la Société Saint-Luc, 27 septembre 1884, reproduit dans Bull. Méd. Soc. S.L., janv. 1927, p. 7.

22.. Ange-Ernest Amédée FERRAND, Intervention à l'Assemblée générale de la Société Saint-Luc, 1886, p. 27, Arch. C.C.M.F.

23.. On perçoit la difficulté pour un médecin de s'afficher en tant que catholique vers 1890 dans le nombre important de refus de faire paraître son nom dans l'annuaire de la Société. Beaucoup de médecins, surtout en province, refusent que le comité directeur développe les opérations publicitaires sur l'extérieur, car la clientèle de cabinet y semble sensible. Ce genre de résistance apparaît moins fort une dizaine d'années plus tard, et surtout dans l'entre-deux-guerres.

24.. Sur les congrès scientifiques catholiques de la fin du XIX ${ }^{\mathrm{e}}$ siècle, et sur Mgr d'Hulst, voir Francesco BERETTA, Monseigneur d'Hulst et la science catholique, portrait d'un intellectuel, Paris, Beauschène, 1996.

25.. Sur Mgr Mercier et les développements du thomisme dans les milieux scientifiques, voir Roger AUBERT, Le cardinal Mercier (1851-1926). Un prélat d'avant-garde, Academia, Louvain, 1994.

26.. La Faculté libre de médecine de Lille a été créée en 1877, par un groupe de médecins qui figurent pour la plupart parmi les fondateurs de la Société Saint-Luc. 27.. Ce bulletin est d'abord trimestriel, puis mensuel. En 1955, il prend le nom de SaintLuc, Évangile et société, puis en 1968 celui de Médecine de l'homme.

28.. Bull. Méd. Soc. S. L., février 1897.

29.. Les premières assemblées générales de la Société se déroulent à l'Institut catholique de Paris, tandis qu'à Lyon, les professeurs de la Faculté sont régulièrement conviés. Plusieurs comités régionaux se réunissent dans des locaux prêtés par des ordres monastiques bénédictins (Marseille) ou franciscains (Rennes).

30.. Requête présentée lors de la réunion du conseil central de la Société Saint-Luc, en juillet 1901, Arch. C.C.M.F. Une requête similaire a lieu en juin 1904.

31.. Procès-verbal de la Société Saint-Luc, juin 1904, Arch. C.C.M.F. Dans ces procèsverbaux, les discussions sont très libres et l'Église n'y apparaît guère comme une instance normative.

32.. Procès-verbal de la réunion du comité parisien de la Société médicale Saint-Luc, mars 1898, Arch. C.C.M.F.

33.. Les réunions de la Société Saint-Luc se déplacent au Cercle du Luxembourg, en 1892.

34.. En 1890, face à une pyramide des âges vieillissante des adhérents, proposition est faite de multiplier les contacts avec les centres universitaires afin d'attirer les jeunes médecins, et pour ce faire, les médecins adhérents qui enseignent dans ces centres, sont chargés de convier les étudiants.

35.. Sur Laënnec, voir Dr Paul MICHAUX, « Les Origines et le passé de la conférence », dans Conférences Laënnec, décembre 1919, pp. 5-46 et Jacalyn DUFFIN, To see with a better eye, a life of R.T.H. Laënnec, Princeton University Press, 1998. 
36.. Henri Dauchez est l'un d'eux, mais le docteur Ange Ferrand, président de la Société Saint-Luc, intervient de façon permanente dans la Conférence entre 1879 et 1899.

37.. «Le Médecin catholique. Mémento de la Conférence Laënnec », dans Conférences Laënnec, mai 1911, pp. 23-24.

38.. En 1899, le corps médical stricto sensu comporte 12000 praticiens (presque 17000 avec les officiers de santé), en 1911 environ 20 000. Le rapport des effectifs de la Société à ce chiffre est donc stable - autour de cinq à six pour cent. Voir Christian BONAH, Instruire, guérir, servir. Formation et pratiques médicales en France et en Allemagne pendant la deuxième moitié du XIX ${ }^{e}$ siècle, Presses Universitaires de Strasbourg, 2000, p. 77.

39.. Entre 1886 et 1899, c'est-à-dire sous la présidence d'Ange Ferrand, phase d'essor de la Société, la totalité des adhérents des comités de l'ouest (Rennes, Nantes, Rouen, Laval, Le Mans, Poitiers) passe de cinquante pour cent des effectifs à trente pour cent. Les adhérents parisiens restent stables autour de vingt pour cent, tandis que la progression la plus forte est celle des adhérents lillois (de dix à vingt pour cent) et lyonnais (de zéro à douze pour cent).

40.. L'élaboration d'un annuaire de la Société permet de préciser les zones de force par département et par ville, cette répartition mettant en valeur les départements du Nord, de la Seine, du Rhône et des Bouches-du-Rhône et les implantations universitaires catholiques.

41.. Pierre GUILLAUME, ouv. cité.

42.. Il s'agit des docteurs Henri Desplats et François Guermonprez.

43.. L'expression n'est pas usurpée, car aucune femme ne fait partie du groupe. Longtemps la Société Saint-Luc s'est posé la question de l'adhésion des femmes et la première qui fut acceptée est le docteur Boudeille en 1912.

44.. Sur la spécialisation médicale, voir Christian BONAH, ouv. cité, pp. 109-116.

45.. Les publications qui marquent l'évolution de sa pensée sur ce point sont, dans l'ordre chronologique : Considérations médico-légales sur la responsabilité des faibles d'esprit (1881), De la séquestration des aliénés dans leurs familles (1884), Relation d'un cas de simulation de la folie (1885), La raison dans la folie, étude pratique et médico-légale sur la persistance partielle de la raison chez les aliénés et sur leurs actes raisonnables (1888). Victor Parant est l'auteur d'une traduction de Hack Tuke, Le corps et l'esprit, action du moral et de l'imagination sur le physique (1886) et d'une étude sur le secret médical et les aliénés, parue dans le Bulletin de la Société Saint-Luc, en 1903.

46.. Le Père Pierre-Jean-Corneille Debreyne (1786-1867) est médecin et moine à la grande Trappe (Orne), élève de la Faculté de médecine de Paris. Il est un auteur très lu dans le domaine de la médecine morale.

47.. Ange-Ernest-Amédée Ferrand (1835-1899) a marqué fortement de son empreinte l'histoire de la première Société Saint-Luc.

48.. Il a des responsabilités dans les sociétés suivantes : Secrétaire général de la Société médicale d'émulation (1868), de la Société de médecine de Louvain, président de la Société de thérapeutique, président de la Société médicale des hôpitaux (1893).

49.. Marcel GAUCHET, L'Inconscient cérébral, Éditions du Seuil, 1992.

50.. Joseph Grasset (1848-1918) est agrégé (1875), interne des Hôpitaux de Montpellier (1871). Il est nommé par le doyen, contre l'avis du ministre Paul Bert, à la chaire de pathologie interne de l'Université de Montpellier, très ancienne université médiévale. Il fait partie du comité de rédaction de la revue L'Encéphale, de Montpellier médical, participe à la fondation des Archives de neurologie, donne des articles aux Annales de psychiatrie et d'hypnologie ainsi qu'à la Revue de l'hypnotisme et de thérapeutique, collabore 
au Dictionnaire encyclopédique des sciences médicales. Il est aussi membre de l'Académie de médecine et de la Société psychiatrique de Paris, Secrétaire général de la Société de médecine et de chirurgie pratique.

51.. Jean Camus (1872-1924), agrégé de la faculté, est psychiatre des établissements publics d'aliénés après avoir exercé à l'hôpital de la Charité. Membre de l'Académie de médecine, il développe ses recherches sur l'hypophyse, puis sur la psychothérapie. Il publie en 1904 un traité en collaboration avec le docteur Pagniez, Isolement et psychothérapie, Paris, F. Alcan, 1904.

52.. Sur l'évolution de la psychothérapie après 1900 on dispose encore de peu d'ouvrages. Voir Jacqueline CARROY, Hypnose, suggestion et psychologie, l'invention de sujets, Presses universitaires de France, 1991.

53.. On peut attribuer cette corrélation à plusieurs facteurs : la médecine catholique s'intéresse à cette question dès l'origine, par inclination naturelle vers le fonctionnement de l'esprit, mais aussi par obligation, afin de répondre aux accusations scientifiques qui pèsent sur les phénomènes mystiques, les apparitions, les possessions, fréquentes après 1880 . On peut aussi voir dans cet intérêt pour ce domaine précis, un écho de l'attrait général pour ces questions entre 1880 et 1930. Pour notre part, nous inclinons à penser que le catholicisme et plus largement le christianisme entretiennent un rapport étroit avec la science des troubles de l'esprit.

54.. Henri Desplats (1843-1912), doyen de la Faculté libre de médecine, est président de la Société scientifique de Bruxelles en 1877. Praticien de l'hypnose, il rédige plusieurs brochures sur la question dans les années 1880.

55.. Ruth HARRIS, ouvr. cité.

56.. Camille Féron-Vrau (1831-1908), médecin lillois, exerce peu son métier et se tourne vers l'action catholique. Il anime l'Association catholique des patrons du nord et est un des mécènes fondateurs de la Faculté libre de Lille, ainsi que de plusieurs hôpitaux du nord et institutions d'accueil.

57.. La répartition thématique des sujets relève d'un choix nécessaire dans ce type d'étude quantitative, choix qui pourrait être différent. Certains regroupements pourront paraître artificiels ; ils permettent cependant de donner une image satisfaisante des préoccupations intellectuelles des hommes de la Société.

58.. Cette comparaison ne peut être qu'approximative au vu du peu d'études sur le monde médical de cette époque.

59.. Concernant le baptême, le recours aux médecins par les fidèles et par l'Église peut paraître incongru ; pourtant l'histoire du christianisme, à travers les travaux de Philippe Ariès notamment, a montré l'importance du baptême des enfants mort-nés pour les parents inquiets.

60.. Concernant le mariage, il s'agit surtout de développer une forme de jurisprudence médicale appliquée aux empêchements. Les médecins peuvent interdire le sacrement ou éventuellement le déconseiller. Les cas d'empêchement des aliénés et des épileptiques sont les plus fréquents, mais on constate une extension de ce questionnement aux tuberculeux et à d'autres pathologies, supposées héréditaires et donc soumises à un contrôle social accru.

61.. Concernant le moment de la mort, les questions posées aux médecins catholiques sont multiples : comment déterminer cliniquement l'imminence de la mort, afin que le prêtre puisse pratiquer l'extrême-onction ? La crémation est-elle légitime dans certains cas, au détriment de l'inhumation, qui reste la règle sociale et religieuse communément 
admise malgré les manifestations de la Libre Pensée. Voir Jacqueline LALOUETTE, La Libre Pensée en France (1848-1940), ouv. cité.

62.. Procès-verbal du comité parisien de la Société Saint-Luc, déc. 1898, Arch. C.C.M.F. ; la même question se pose à propos du cas des épileptiques en 1899. De façon générale, toute pathologie est montrée comme un handicap matrimonial, qu'il s'agisse de la tuberculose, de la syphilis ou de la maladie mentale.

63.. Dans l'ordre chronologique des périodes étudiées : Henri ELLENBERGER, Histoire de la découverte de l'inconscient, Paris, Librairie Arthème Fayard, 1994 ; Jacqueline CARROY; ouv. cité ; Nicole EDELMAN, Voyantes, guérisseuses et visionnaires en France, Paris, Albin Michel, 1995 ; Marcel GAUCHET, ouv. cité ; R. PLAS, Naissance d'une science humaine, la psychologie, Rennes, Presses Universitaires de Rennes, 2000 ; Elisabeth ROUDINESCO, Histoire de la psychanalyse en France, vol. 1, 1885-1939, Paris, Librairie Arthème Fayard, 1994.

64.. Allocution du Dr Ferrand pour l'assemblée générale du 21 octobre 1888, reproduite dans Société médicale Saint-Luc, Saint-Côme et Saint-Damien, Assemblée générale, Lille, 1889, p. 31, Arch. C.C.M.F.

65.. Sur l'importance des conceptions hygiénistes dans le monde médical et dans l'ensemble de la Société : Alain CORBIN, Le Miasme et la jonquille. L'odorat et l'imaginaire social, $18^{e}-19^{e}$ siècles, Paris, Aubier, 1982 ; Georges VIGARELLO, Histoire des pratiques de santé. Le sain et le malsain depuis le Moyen-Age, Paris, Éditions du Seuil, 1999 ; Pierre DARMON, L'Homme et les microbes, XVII ${ }^{e}$-XXe siècles, Paris, Librairie Arthème Fayard, 1999.

66.. Martial SEVEGRAND, ouv. cité, pp. 40-46.

67.. Les dix-huit apparitions de Lourdes relatées par Bernadette Soubirous ont lieu à partir de février 1858, et ont été étudiées dans de nombreux ouvrages : René LAURENTIN, Lourdes, documents authentiques, Paris, Lethielleux, 1957-1965 ; Joachim BOUFLET et Philippe BOUTRY, «Un Signe dans le ciel » : les apparitions de la Vierge, Paris, Éditions du Cerf, 1997, pp. 151-158; Ruth HARRIS, ouv. cité.

68.. Yves CHIRON, Enquête sur les miracles de Lourdes, Paris, Perrin, 2000, pp. 64-77 ; Pierre GUILLAUME, Médecins, Église et foi, XIX ${ }^{e}-X X^{e}$ siècles, Paris, Aubier, 1990, pp. 63-64 ; l'information la plus précise et la plus proche des sources est donnée par Ruth HARRIS, ouv. cité, pp. 430-443.

69.. Le docteur Dunot de Saint-Maclou, dont la thèse sur l'avortement fut remarquée par l'Université de Louvain, est amené à fréquenter le sanctuaire pour sa femme, malade depuis 1887. Il s'installe en 1883, probablement sur la demande des frères assomptionnistes, et développe par la suite ses études médico-théologiques. C'est peu après son décès, en 1891, que le docteur Gustave Boissarie (1836-1917) prend le relais à la présidence du Bureau. Interne des Hôpitaux de Paris, il effectue l'essentiel de sa carrière comme chirurgien à Sarlat (Dordogne) avant d'être appelé à la direction du Bureau de constatation médicale de Lourdes en 1892. Ses publications sont centrées uniquement sur le sanctuaire et ses guérisons : Lourdes, Histoire médicale (1891), Les Grandes guérisons de Lourdes (1900), Les Guérisons (1911). Sur le sujet, il publie plusieurs articles dans le Bulletin Médical de la Société Saint-Luc et répond aux écrits de Zola par des conférences dans toute la France, et notamment au Cercle du Luxembourg. Le docteur Edouard Le Bec, membre de la Société Saint-Luc depuis l'origine, est directeur du comité parisien et président du conseil national en 1904. Ce spécialiste de chirurgie obstétrique exerce à l'hôpital Saint-Joseph. À partir de 1892, il s'intéresse à la question des guérisons de Lourdes et publie par la suite plusieurs ouvrages et brochures : Preuves 
médicales du miracle (1917); Critique et contrôle médical des guérisons surnaturelles (1920); Lourdes et la Franc-maçonnerie (1938).

70.. On compte moins de trente médecins sur les lieux en 1890, la plupart catholiques ; en 1913, on en compte plus de cinq cents partagés entre catholiques, anticléricaux, simples curieux, et plus de deux cents confrères étrangers.

71.. La création du Bureau de constatation conduit à la diminution brutale des guérisons reconnues en 1884 : de cent quarante-trois à quatre-vingt-trois. Chiffres donnés par Ruth HARRIS, d'après les Annales de Notre-Dame de Lourdes, volume 16 (1883-1884) et Théodore MANGIAPAN, Les guérisons de Lourdes, Lourdes, Euvres de la grotte, 1994.

72.. Un modèle de ce rapport médical figure dans le Bull. Méd. Soc. S. L. , novembre 1911. Celui-ci détaille les affections et notamment l'ensemble des pathologies névropathiques possibles.

73.. Le débat sur la création d'une commission d'enquête et sur l'établissement de certificats commence dès 1891 ; Société Médicale De Saint-Luc, Saint-Côme et SaintDamien, Assemblée genérale du 18 octobre 1891, Paris, 1892, Arch. CCMF. L'année suivante, les assomptionnistes, qui sont les maîtres d'œuvre du pèlerinage, s'adressent à la Société pour lui demander de visiter à domicile les pèlerins avant leur arrivée. 74.. Le comité du Sud-Ouest, d'abord centré sur Toulouse, se développe après 1903 : il passe d'une quinzaine de membres à vingt-cinq. En 1904 est créé sous l'impulsion du docteur Cox, adjoint du docteur Boissarie, un comité de Lourdes.

75.. Ce référendum lancé par Henri Dauchez reçoit près de trois mille réponses favorables au maintien des bains du sanctuaire. Bulletin médical de la Société Saint-Luc, Saint-Côme et Saint-Damien, novembre 1906, p. 217.

76.. Louis Van der Elst, membre de la Société Saint-Luc (président du comité parisien en 1922) et des Conférences Laënnec, enseigne à l'Institut catholique de Paris. Il est spécialiste des névroses et de la neurasthénie.

77.. La médecine neurologique et psychiatrique des années 1880-1910 remet en cause la religiosité des états mystiques, le plus souvent au nom du diagnostic hystérique. Les phénomènes de stigmatisation, c'est-à-dire de reproduction des stigmates sanglants du Christ, sont ainsi montrés comme des simulations ou comme des cas d'idéoplastie résultant d'une pathologie nerveuse. Sur l'interprétation hystérique des mystiques et sur les phénomènes de stigmatisation : Nicole EDELMAN, Les Métamorphoses de l'hystérique, du début du XIX ${ }^{e}$ siècle à la Grande guerre, Paris, La Découverte, 2003 ; Jacques MAITRE, Les Stigmates de l'hystérique et la peau de son évêque. Laurentine Billoquet (1862-1936), Paris, Anthropos, 1993 ; Elisabeth ROUDINESCO [dir.], Autour des études sur l'hystérie, Vienne 1895, Paris 1995, Paris, L'Harmattan, 1998 ; Pierre-Henri CASTEL, La Querelle de l'hystérie, Paris, Presses universitaires de France, 1998 ; Joachim BOUFLET, Les Stigmatisés, Paris, Éditions du Cerf, 1996 ; Dominique De COURCELLES [dir.], Stigmates, Paris, L'Herne, 2001.

78.. René LE FUR, « Lourdes et les médecins », dans Bull. Méd. Soc. S. L., déc. 1901, pp. 177 et 183. L'auteur fait de multiples recommandations pour développer l'approche scientifique de la critique des guérisons miraculeuses : certificats établis avant le pèlerinage, diffusion de l'usage de la photographie et de la radiographie, etc.

79.. Les médecins du pèlerinage ne sont pas autorisés à se rendre dans les piscines, et ne peuvent pénétrer dans les salles des malades sans l'autorisation des sœurs qui dirigent les services. Les récits de guérison sont souvent établis grâce au témoignage 
des brancardiers. Enfin, les médecins qui examinent le malade avant et après la guérison ne sont jamais les mêmes.

80.. Le président Gouraud affirme ainsi que Boissarie « se défend de certaines critiques à l'égard des médecins catholiques qui feraient preuve d'une obstruction volontaire systématique. Bien des médecins catholiques et lui en particulier ont adressé des observations qui ont été mal accueillies en ce qui concerne le contrôle des miracles et la rigueur scientifique que l'on doit apporter dans leur vérification. Quoi qu'il en soit, les réserves de monsieur Le Fur sont absolument fondées ». Procès-verbal de la réunion du comité parisien de la Société Saint-Luc, juin 1901. L'ensemble du comité parisien applaudit les conclusions favorables au docteur Le Fur.

81.. Sur le roman de Zola, Lourdes, paru en 1894, ou sur celui de Huysmans, Les Foules de Lourdes, paru en 1906, romans qui s'opposent quant à la représentation des lieux, voir Ruth HARRIS, ouv. cité, pp. 444-454.

82. Le Pèlerin, 9 décembre 1894.

83.. On peut citer plusieurs exemples. Charcot ne rejette pas en bloc les miracles modernes, mais en connaît la portée ; sur la fin de sa vie, en 1893, il publie un article, $L a$ Foi qui guérit, qui témoigne d'une pensée complexe. Pierre Janet, psychologue éminent, réhabilite les pratiques de l'exorcisme dans son approche du cas d'Achille. Citons aussi les processions de l'asile laïc d'aliénés du département de l'Orne, destinées à procurer une émotion thérapeutique aux malades.

84.. Le nombre d'adhérents dépasse le millier après 1910.

85.. Les comités de l'Ouest (Nantes, Rennes, Rouen, Laval, Le Mans) représentent plus d'un adhérent sur trois dans les premières années de la Société et n'en représentent plus qu'un quart en 1908, alors que la croissance des effectifs reste forte à cette époque. On peut attribuer cette diminution relative au manque de dynamisme du recrutement de comités anciens dirigés par des présidents faisant partie des fondateurs de la Société. On peut aussi n'y voir qu'un effet du rattrapage des autres régions : progression de l'Est et du Sud. On peut enfin distinguer dans cette évolution un effet de l'orientation nouvelle donnée par les dirigeants de la Société après 1900, qui impliquerait une évolution distincte entre régions catholiques traditionnelles et nouvelles régions acquises.

86.. En 1904, un curé du diocèse d'Orléans, l'abbé Guerre, qui fonde une œuvre pour les enfants arriérés, s'adresse directement à la Société pour lui recommander quelques praticiens afin de développer son activité.

87.. L'une des raisons de ce pèlerinage réside dans la volonté de s'appuyer sur les guérisons miraculeuses afin de convertir une partie du monde médical. Pour les organisateurs : « Les faits vont se multipliant, et l'on trouverait sans doute peu de médecins en France qui n'aient été, à un moment de leur carrière, avant ou après les pèlerinages, aux prises avec un ou plusieurs faits merveilleux de Lourdes, et mis ainsi, parfois même malgré eux, en face de ces touchantes manifestations surnaturelles ", dans Bull. Méd. Soc. S. L., janvier 1904, p. 29.

88.. La Société Saint-Luc de France compte, en 1908, 46 membres étrangers, dont la plupart sont belges (10) et canadiens (13), les autres se répartissant principalement entre l'Italie, la Suisse, les Etats-Unis, le Danemark, l'Espagne et le Brésil. D'autres sociétés médicales sont apparentées à celle de Saint-Luc comme la Societa medica cattolica sotto la protezione di san luca, fondée en 1904, qui prend le relais d'une société plus ancienne fondée en 1897 à Milan, la Societa medica S. Antonio Maria Zaccaria. Une société est fondée à Florence en 1904, sous l'impulsion d'un membre franciscain de la 
société française, le docteur Longo. En Angleterre, la Guild of S. Luke, S. Cosmas and S. Damien, fondée en 1910, compte 166 membres en 1911, soit près de sept fois moins d'adhérents que la société française. La société hollandaise est fondée en 1905 avec 150 membres, en association avec la création d'une faculté libre de médecine. La société belge de Saint-Luc s'organise en 1906 tandis qu'existe depuis 1875 la Société scientifique de Bruxelles, qui compte 75 membres en 1903 et édite depuis 1877 la Revue des questions scientifiques, qui jouit d'une audience jusqu'en France. En Espagne la Société médico-pharmaceutique de Saint-Côme et Saint-Damien, créée en 1884 sous l'impulsion de pères jésuites, est sise à Barcelone et compte 144 membres en 1905 ; elle publie une revue mensuelle : Le Jugement catholique dans les sciences médicales. Au Danemark, l'impulsion vient aussi d'un missionnaire français, le docteur Piou de SaintGilles, rédemptoriste, qui crée en 1904 un petit groupe de médecins catholiques. C'est la même année qu'est fondée l'Association médicale de Saint-Raphaël et Saint-Luc de Rio de Janeiro.

89.. Sur les premiers congrès internationaux de médecine catholique, voir J. LIEFFOGHE, art. cité, p. 128 ; Pierre GUILLAUME, Médecins, Église et foi, XIX ${ }^{e}$-XXe siècles, ouv. cité, p. 111.

90.. Bull. Méd. Soc. S. L., novembre 1905, p. 177.

91.. La menace de scission interne est réelle sur la question de la nature de l'organisation. En 1904, la majorité des médecins catholiques refusent la transformation syndicale de la Société, au bénéfice de l'affiliation à des organisations interprofessionnelles dont certaines sont dirigées par des médecins de la Société SaintLuc.

92.. Lors de l'assemblée de 1907, une vingtaine de membres de la Société appuie une révision des statuts conforme à la loi de 1901 sur les associations. Une grande majorité de comités est favorable à la déclaration légale de la Société, et une première mouture des statuts rénovés ne mentionne pas l'appartenance religieuse des membres. Finalement celle-ci est rétablie devant la réticence d'une partie des adhérents.

93.. Le débat est retranscrit dans les procès-verbaux du comité parisien de la Société Saint-Luc, juin 1903, Arch. C.C.M.F.

94.. Parmi ceux-ci les plus diffusés sont ceux de LAVRAND et MOURAU, Le Médecin chrétien, Paris, Lethielleux, 1905 ; Georges SURBLED, La Morale dans ses rapports avec la médecine et l'hygiène, Paris, Retaux, 1900 ; Henri BON, Précis de médecine catholique, Paris, Alcan, 1935.

95.. François LAPLANTINE, Anthropologie de la maladie, étude ethnologique des systèmes de représentations étiologiques et thérapeutiques dans la société occidentale contemporaine, Paris, Payot, 1986.

96.. Voir par exemple Jack D. ELLIS, The physicians-legislators of France : medecine and politics in the early Third Republic, 1870-1914, Cambridge University Press, 1990. L'auteur tempère par une approche sociologique l'anticléricalisme des professions médicales. 97.. Michel LAGRÉE, La Bénédiction de Prométhée. Religion et technologie, Paris, Fayard, 1999.

98.. Autour des Études carmélitaines, qui paraissent à partir de 1933, et du Père Bruno de Jésus Marie, se sont associés ainsi médecins, psychiatres et hommes d'Église afin d'aborder des questions épineuses au sein de l'Église telles que les phénomènes de stigmatisation (Thérèse Neumann), d'apparitions (Beauraing) ou encore l'ensemble des aspects de la mystique. Plusieurs médecins de la Société Saint-Luc s'associent à cette réflexion. 


\section{RÉSUMÉS}

La Société médicale Saint-Luc, Saint-Côme et Saint-Damien est créée en 1884 par des médecins catholiques, à des fins apologétiques et scientifiques, à l'époque de la construction de la République laïque. L'étude sociologique, géographique, statistique et institutionnelle de ce groupe, montre que les praticiens catholiques ne se distinguent que faiblement de leur corps professionnel et présentent des visages variés. Ils favorisent l'éclosion d'une science originale fondée sur une morale et une doctrine, à des fins de défense ou de conversion, parfois mise au service de l'Eglise, lorsqu'il s'agit par exemple de défendre le sanctuaire de Lourdes, parfois indépendante. Le concept de "médecine catholique", en débat à cette époque, doit être interrogée par les historiens. Le médecin chrétien, figure contradictoire de la fin du XIX ${ }^{\mathrm{e}}$ siècle, paraît jouer un rôle important dans le processus de sécularisation de la société et dans ce que nous appelons aujourd'hui éthique.

The beginnings of Catholic medicine in France. The medical society of Saint-Luc, SaintCôme and Saint-Damien (1884-1914). The medical society of Saint-Luc, Saint-Côme and SaintDamien, was created in 1884 by Catholic doctors to promote both their faith and scientific knowledge, during the foundation of a Republic separated from the Church. A sociological, geographical, statistical and institutional study of this group shows that Catholic practitioners were only slightly different from their non religious peers and may have different faces. They contributed to the creation of a particular science grounded on morals and doctrine aimed at defending or promoting their faith. They either stayed independent or occasionally spoke out for the Church, for instance to defend the Lourdes sanctuary. The concept of a "Catholic science of medicine", as it was debated at this time, should be questioned by historians. The Christian doctor was a contradictory figure in the late $19^{\text {th }}$ century, but seemed to play a great role in the secularisation process of society, and in what is nowadays called ethics.

\section{AUTEUR}

HERVÉ GUILLEMAIN

Agrégé, doctorant à l'Université Paris XII 